
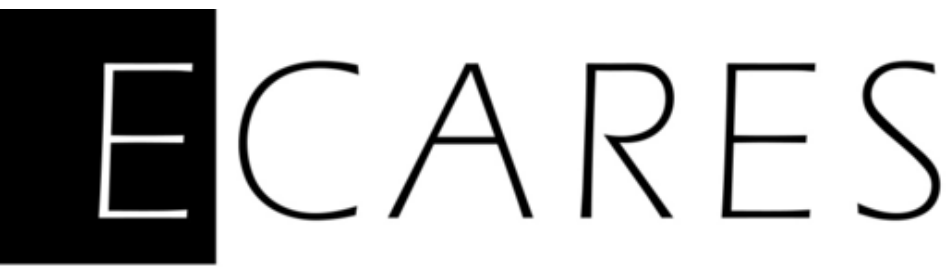

\title{
It Does (not) Get Better: \\ Expected Income Violation and Altruism
}

Julien Benistant

ISC-CNC, CNRS UMR

Rémi Suchon

ECARES, Université libre de Bruxelles

August 2020

ECARES working paper 2020-35 


\title{
It does (not) get better: expected income violation and altruism.*
}

\author{
Julien Benistant ${ }^{\dagger}$ - Rémi Suchon ${ }^{\ddagger}$
}

We experimentally test whether the gap between expected and actual income impacts subsequent altruism. Participants first perform a real-effort task for a fixed wage and then play a dictator game. Between conditions, we vary the level and the timing of the revelation of the wage. In some conditions, participants know the wage before the real effort task and are not informed of the other potential levels. In some other conditions, they are informed of the distribution of wages before the real effort task, but the actual wage is only revealed afterward. Participants in the latter conditions can form expectations that may be higher or lower than their actual wage. Our model predicts that the gap between the expected and the actual wage impacts transfers in the subsequent dictator game. The results support this hypothesis: participants who get the low wage transfer less and are less likely to transfer when they are informed of the other potential levels than when they are not. Conversely, participants who get the high wage are more likely to transfer positive amounts when they are informed of the other potential levels. We use physiological (skin conductance response) and declarative data to discuss the role of emotions in our treatment effects.

*The authors are grateful to audiences at CREED (Amsterdam), GATE (Lyon), ECARES (Brussels), IESEG (Lille), the 6th IMEBESS (UTRECHT) \& the 10th ASFEE conference (Toulouse). This paper benefited from discussions with Brice Corgnet, Jan Engelmann, John Hamman, Astrid Hopfensitz, Nicolas Jacquemet, Agne Kajackaite, Mathieu Lefebvre, Benoît Tarroux, Chloé Tergiman, Marie Claire Villeval and Marc Willinger. Mateus Joffily and Quentin Thevenet provided valuable technical assistance. Julien Benistant thanks the Fondation pour la Recherche Médicale for financial support (FDT20170436847). This research was funded by the LABEX CORTEX (ANR-11-LABX-0042) of Université de Lyon, within the program "Investissement d'Avenir" (ANR-11-IDEX-007) operated by the French National Research Agency (ANR). It received the approval of the Gate-lab research Ethics Committee (ref.: 2018-12).

†ISC-CNC, CNRS UMR 5229. 67 Boulevard Pinel, 69500 Bron, France. julien.benistant@isc.cnrs.fr

${ }^{\ddagger}$ ECARES, Université Libre de Bruxelles. Avenue F. D. Roosevelt 50, CP 114, B-1050 Brussels, Belgium. remi.suchon@ulb.ac.be 


\section{Introduction}

People form expectations about the outcome of uncertain processes of importance to them, but these expectations are often violated. People form expectations about their income, but unforeseeable shocks may make one better or worse off than expected, for instance because of a sudden change in their firm's wage policy, a macro-economic slowdown or a pandemic. Alternatively individuals themselves may make mistakes in projecting the income they would get due to overconfidence, the lack of relevant information or cognitive limits. The gap between the actual and expected outcome of these processes have an autonomous impact on their behavior. For instance, when the chance to earn a high income increases (setting high expectations), people tend to lie more to avoid a low income (Garbarino et al., 2019), the defeat of a football team is more likely to trigger family violence in the fans when they were expecting a victory (Card and Dahl, 2011) and lower price expectations reduce consumers' willingness to pay for a good (Heidhues and Köszegi, 2008).

In this paper, we study how expectations about income affect social preferences. More precisely, we ask: what is the impact of the gap between expected and realized income on altruism? Does an individual who gets a lower income than expected compensate the gap between his expectations and the realization by becoming less generous? Symmetrically, does receiving an income greater than expected translate into higher generosity? To illustrate what we have in mind, consider an individual who contemplates giving money to a friend in need: would his likelihood of doing so depend on whether his recent income differs from his expectations? A possibility is that, if his income is lower than expected, he might be reluctant to give because he wants to reduce the gap between his expectations and his realized income. This is the case if his expectations are used as references: falling below the reference typically triggers reactions to reduce the feeling of loss. We can also think of an individual who is asked to give to a charity at the exit door of a grocery store: would he be more willing to give if he obtained unexpected rebates on the basket he had planned to buy? In this case, he might feel that the money he saved exceeds his reference point, and thus be willing to share it because he does not feel strongly attached to it.

We focus on the pure effects of expectations violation, controlling for the actual income. Throughout our analysis, we hold the set of achievable distributions of income and intentions constant, so that outcome-based (e.g. Fehr and Schmidt, 1999; Bolton and Ockenfels, 2000) or intention-based (Rabin, 1993; Dufwenberg and Kirchsteiger, 2004) theories of social preferences do not provide specific predictions on the effect of expected wage violations on altruism. However, if, as we hypothesize, expectations constitute a referent to which individuals compare their actual income, then expectations violations may have an autonomous impact on altruism: individuals might modulate their generosity depending on the gap between their expected and actual income, because they define gains and 
losses relative to expectations. When one receives an income that is lower than expected, one might want to tighten the gap by becoming less generous, while an individual who receives an higher income than expected might be willing to share more because he does not feel strongly attached to the income he was not expecting.

To isolate the pure effect of expectations violation on altruism, our laboratory experiment manipulates independently wages and wage expectations controlling for income effects. In the first part, participants have to perform a real effort task in exchange of a fixed wage. In the second part, they play a dictator game and they have to decide how much of a fixed endowment they are willing to transfer to an other anonymous participant. In a $3 \times 2$ between-subjects design, we vary the wage for the real effort task (low, medium, high) and the timing of information on the wage (Ref and NoRef). In the NoRef conditions, participants are informed upfront of the wage in the real effort task and they are given an envelope containing the corresponding wage before performing the real effort task. In the Ref conditions, they only know the distribution of potential wages and they are informed that the actual wage will be revealed after the real effort task. In all Ref conditions, participants receive an envelope containing the medium wage from which we take out money in the $R e f_{\text {low }}$ condition or add money in the $R e f_{\text {high }}$ condition after the revelation of the actual wage. By informing participants of the distribution of the wages, we let them form expectations, that are higher (resp. lower) than their actual wage when they receive the low (resp. high) wage. To isolate the pure effects of expectations, we compare transfers in the Ref conditions to the transfer in the NoRef conditions, for each level of wage.

In our task, we expect that participants compare their actual income to an expectation based reference point. An income different from expectations may induce an emotional arousal associated with the experience of losses or gains. Previous evidences suggest that the behavioral effects of losses and gains are channeled by emotions (Card and Dahl, 2011; Ring and Schmidt, 2019). In turn, emotions have been shown to carry over to subsequent decisions (e.g Loewenstein and Lerner, 2003; Lerner et al., 2015) for instance influencing pro-sociality and altruism (Capra, 2004; Kirchsteiger et al., 2006; Andrade and Ariely, 2009). Anger and frustration are also suspected to affect subsequent social behavior (Drouvelis and Grosskopf, 2016; Battigalli et al., 2019) although recent experimental evidences are mixed (Persson, 2018). In our experiment, emotions such as elation or disappointment induced by expected wage violation may channel our treatment effects. For instance, we may expect that receiving a wage lower than expected (a negative expectation violation) may trigger negative emotions and affect the subsequent transfers. We collected data on the participants' emotional state throughout the experiment, with both physiological (Skin Conductance Response) and declarative measures (Bosman and van Winden, 2002). Using this data, we can directly test whether reference violations 
trigger emotional responses, and whether these responses impact behavior, contributing to our understanding of reference dependent preferences.

In order to theoretically explore how expected income violation could impact transfers in the dictator game, we develop a model of sharing with reference-dependence (Kahneman and Tversky, 1979; Tversky and Kahneman, 1991; Köszegi and Rabin, 2006). A participant forms (potentially biased) expectations about his payoff for the whole experiment based on the information he receives at the beginning of the experiment and then evaluates his actual payoff relative to his expectations. An important feature of our model is that we do not need to assume different concerns for the recipient's payoff across the gain and the loss domains. Yet, the model predicts that participants in the $R e f_{\text {low }}$ condition will transfer less than those in the $N_{o R e} f_{\text {low }}$ because it is a way for them to reduce the gap between their expected payoff and the payoff they actually get. The model also predicts that participants in the $R e f_{\text {high }}$ condition will transfer more than those in the NoRe $f_{\text {high }}$ condition.

The experimental data provide some support to the theory. Transfers are lower in $R e f_{\text {low }}$ compared to NoReflow, both at the intensive and at the extensive margins. For the high wage condition, results are more nuanced as average transfers are not significantly different between Re $f_{\text {high }}$ and NoRe $f_{\text {high }}$. This suggests that "losses loom larger than gains" (Kahneman and Tversky, 1979). Nonetheless, null transfers are less frequent in $R e f_{\text {high }}$ than in NoRe $f_{\text {high }}$. In both cases, the effects are larger for loss averse participants. This provides supports to the idea that expectations are used as reference, and that reference violations have an independent effect on altruism. We also find that participants transfer similar amounts in the $R e f_{\text {med }}$ and NoRe $f_{\text {med }}$ conditions. Turning to emotions, we find that participants in the Ref conditions have greater physiological responses than in the NoRef conditions. In addition, participants in the $R e f_{\text {low }}$ declare more negative emotions while participants in the $R e f_{\text {high }}$ condition declare more positive emotions than in the respective NoRef conditions. Furthermore, a higher physiological response predicts a higher impact of expected income violation in the $R e f_{\text {low }}$ conditions but not in the $R e f_{\text {high }}$ ones. This is consistent with our behavioral results showing an asymmetry between the effect of losses and gains. Further analysis suggests that anticipatory emotions, measured just before the revelation of the actual wage, predicts transfers better than expost emotions. This provides insights into the role of emotions in reference dependent preferences.

Section 2 reviews the relevant literature, section 3 describes the experimental design and procedures, Section 4 introduces the theoretical framework and the hypotheses tested in the experiment, Section 5 presents the results and Section 6 concludes. 


\section{Literature review}

We contribute to three streams of the literature. First, our results join a broad literature showing that subtle manipulations pertaining to the context of the decision rather than to its potential consequences impacts other regarding behavior in a consistent and predictable way (e.g. Dana et al., 2007; List, 2007; Bardsley, 2008). Our experiment relates to this literature because we only manipulate the information about the existence of other levels of wage, varying the context of the decision and keeping unchanged the possible consequences. In doing so, we identify a new contextual factor that potentially impacts one's other-regarding behavior, and provide a theoretical account for it based on a well established conceptual framework: reference-dependent preferences.

Second, by identifying a trade-off between the altruistic preference to give and the selfish preference to avoid private losses relative to an expectation-based reference, our results contribute to a more limited literature that studies social preferences in the loss domain. Some papers have identified that people tend to be more demanding when bargaining over losses than over gains (Buchan et al., 2005; Breitmoser and Tan, 2013; Neumann et al., 2017, 2018), but strategic considerations and social preferences may interact in such settings. In this paper, we mute strategic considerations to focus on social preferences. ${ }^{1}$ Buffat (2016) finds that transfers in his lab experiment are not influenced by the level of an exogenous, randomly implemented transfer. This contradicts the predictions of his expectation-based reference dependent model of social preferences. In contrast, we show that the gap between expected and realized income experienced before the decision to share impacts participants' willingness to share. Thunstrom (2019) and Cochard et al. (2020) study the behavior of participants in dictator games in which the dictator shares either losses or gains. They find evidence of greater altruism in the loss domain. Cochard et al. (2020) further show that this effect is mainly driven by women. The given interpretation is that participants follow a do-no-harm principle: they are reluctant to impose losses to others. In contrast, in our experiment dictators can never explicitly impose losses on receivers which can explain our seemingly opposite results. Boun My et al. (2018) and Fiedler and Hillenbrand (2020) find that participants are less likely to choose the altruistic option in binary dictator games when the payoffs are framed as losses than when payoffs are framed as gains. Using eye-tracking data, Fiedler and Hillenbrand (2020) further show that participants focus their attention on their own outcome more in the loss domain than in the gain domain. In the aforementioned studies, negative payoffs (negative framing) are used to induce losses, while we induce losses by manipulating the gap between actual and expected payoffs without resorting to negative payoffs. Thus, in our experiment a gain or a loss precedes the altruistic decision. In a closely related study, Antinyan (2014) finds that a negative expected income violation does not impact other

\footnotetext{
${ }^{1}$ Breitmoser and Tan (2013) define losses relative to a reference point based on expected payoff, which relates closely to our design.
} 
regarding preferences in the dictator game. The gap with our results may be explained by the fact that only a very small subset of participants are paid for their decisions in the dictator game in this study, making the decisions mostly hypothetical. Another contribution to this literature is that we are able to directly assess the role of emotions in the effects of gains and losses on social preferences. Indeed, emotions have been shown to affect behavior (e.g., Rick and Loewenstein, 2008; Lerner et al., 2015). However, only a few papers have investigated how emotions affect pro-social behavior, using mostly mood or abstract emotional induction procedures (e.g., Capra, 2004; Kirchsteiger et al., 2006; Tan and Forgas, 2010; Ibanez et al., 2017; Pérez-Dueñas et al., 2018). Here, we do not study the effect of emotion on pro-social behavior but rather how emotions can explain the effect of the violations of expectations on a subsequent pro-social behavior. More closely related to our study, Matarazzo et al. (2020) find that negative (positive) expected income violations negatively (positively) affect transfers in the dictator game but that positive and negative emotions does not importantly mediate this effect. Unlike our study, they only rely on declarative emotions and choices are not incentivized.

Last, because we study how individuals respond to receiving a lower (or higher) wage than expected for a real effort task, our paper also relates to the literature on expected wage violation. Kube et al. (2012) recruit participants for a clerical task. Some of the workers are paid less than the wage advertised in the job posting, inducing expected wage violation. They find that these workers provide less efforts. Similarly, Ockenfels et al. (2015) find that receiving a bonus lower than expected reduces both job satisfaction and performance of managers in a large international firm. They confirm these results by means of a controlled lab experiment. In both study, the interpretation is that those who undergo the wage violation negatively reciprocate to those who set the wage. In our study, we mute reciprocity concerns to focus on the effects of falling below or exceeding the expected wage on resource sharing. This corresponds for instance to situations in which the wage cut or the bonus is due to purely exogenous event (e.g. the conjuncture) or the recipient is a colleague rather than the principal. As such, we identify a new potential detrimental effect of wage cuts.

\section{Experimental design and procedures}

\subsection{Experimental game}

Our experimental game consists of three parts. In the first part, participants had to perform a real effort task and in the second part, they played a dictator game. ${ }^{2}$ Instructions

\footnotetext{
${ }^{2}$ In the third and final part, participants were also asked to estimate the average transfer in their session. They were paid $€ 1$ if their prediction was equal to the actual average transfer, plus or minus $€ 0.1$. This data are not analysed in this paper but can be provided upon request.
} 
are in Appendix A.

\section{The real effort task}

In the real-effort task, participants had to encrypt a letter into a number 120 times, with the help of a correspondence table displayed on the computer screen (see the screenshot in Appendix B). For each new letter, the correspondence table changed, and was randomized at the individual level. If the answer was not correct, they had to enter a new number. Thus, every participants had to solve correctly 120 encryption tasks. We chose this real-effort task because it is both easy to explain and tedious. We fixed the number of letters such that most participants would complete the task in 8 to 10 minutes.

For this task, participants were paid a wage of 5, 10 or 15 euros, depending on the experimental condition. Every participants in a session received the same wage. Participants received an envelop containing either one, two or three $€ 5$ banknotes. The envelops were disposed in the cubicles before the session. Our experimental conditions varied the level and timing in which participants were informed of their actual wage for this first part.

\section{The dictator game}

In the second part, participants played a dictator game. All participants had to decide how to share a $€ 5$ endowment with another anonymous participant from the same session. Participants could give any amount between 0 and 5 euros, in increments of 0.1 euro. Participants were informed that they would be randomly matched at the end of the session with another participant. Within each pair, the decision of one participant would be implemented, while the other participant would be the receiver. This procedure enabled us to collect the transfer decisions of every participants in an incentive-compatible way. The transfer decisions in this part constitute our main variable of interest.

\subsection{Experimental conditions}

Every participants in a given session were in the same condition. The experimental conditions vary along two dimensions. Both dimensions are related to the wage for the real effort task. The first dimension varies the level of the wage itself. Participants earned $€ 5$ in the low conditions, $€ 10$ in the medium conditions and $€ 15$ in the high conditions.

The second dimension varies the timing in which participants were informed of their actual wage. In the NoRef conditions, participants were informed of their actual wage before the real effort task. The envelops placed in the cubicles before the session contained their actual and definitive wage. Participants were only informed of the wage they get and that every participants in the session got the same wage, but were not informed 
that other participants in other sessions could get different wages.

In contrast, in the Ref conditions, participants were told at the beginning of the first part that they could earn a wage of either $€ 5, € 10$ or $€ 15$ with equal probability $\left(\frac{1}{3}\right)$. They were informed that the actual wage was determined for each session before the first session of the experiment, that every participants in the session would receive the same wage, and that they would learn their actual wage at the end of the real effort task. The envelops placed in the cubicles before the session contained two $€ 5$ banknotes. In the $R e f_{\text {low }}$ condition, an experimentalist withdrew one $€ 5$ banknote from the envelops after the actual wage had been revealed to subjects. In the $R e f_{\text {high }}$ condition, an experimentalist added one $€ 5$ banknote. ${ }^{3}$ In the Ref conditions, participants could expect a wage different from the wage they would actually get. Presumably, the actual wage would be lower than their expectations in the $R e f_{\text {low }}$ condition, and greater in the $R e f_{\text {high }}$ condition.

To measure our treatment effects, we compare transfers in the Ref and NoRef conditions for each level of wage. This allows to tightly control for potential wealth effects. By comparing transfers in $R e f_{\text {low }}$ and in $N o R e f_{\text {low }}$, we isolate the effect of a negative gap between expected and actual wage. By comparing the transfers in $R e f_{\text {high }}$ and in NoRe $f_{\text {high }}$, we isolate the effect of a positive gap between expected and actual wage.

\subsection{Additional measures}

\subsubsection{Measures of emotions}

In order to assess the potential role of emotions as a channel of the behavioral effects we are investigating, we use two complementary measures of emotional arousal.

We recorded skin conductance (SC), a measure of electro-dermal activity that proxies participants' emotional and physiological arousal (Bradley and Lang, 2000). We applied two electrodes on each participants' non-dominant hand before the start of the experiment and used BioPac ${ }^{T M}$ to measure the electro-dermal activity throughout the experiment. As our interest lies in the participants' emotional arousal at the time of the revelation of the wage in the Ref conditions, we analyzed participants' skin-conductance responses (SCRs) when they were informed of their actual wage. As a control we also analyzed the SCRs of participants' in the NoRef conditions when they were reminded their wage. Precisely, the SCRs were collected during a twelve seconds window. In the NoRef conditions this time window starts from the display of the reminder. In the Ref condition

\footnotetext{
${ }^{3}$ We acknowledge that the mere presence of the experimenter in the room to withdraw or add a banknote in the envelops in the $R e f_{\text {low }}$ and $R e f_{\text {high }}$ may affect behavior. To mitigate this, an experimenter was also present in the experimental room at the time of the reminder in the NoRef conditions and at the time of the wage revelation in the $R e f_{\text {med }}$ condition.
} 
this time window starts 2 seconds before the participants' decision to display their actual wage level up to 10 seconds after this event. ${ }^{4}$ It allows to capture any potential anticipatory reactions as well as the participants' SCRs during the revelation of their wage.

We use a specific procedure, described in Appendix H, to extract the participants' SCRs signal specific to the events taking place during the aforementioned time window. This gives us a value referred to as ISCRs which stands for Integrated SCRs which is often used in experiments with SCRs (e.g., Sokol-Hessner et al., 2009; Ring and Schmidt, 2019).

Recording electro-dermal responses allows to detect any, conscious or unconscious, emotional changes. However, it is uninformative about the valence or the type of emotions experienced by participants. Our second measure of emotions is based on a declarative assessment adapted from Bosman and van Winden (2002) that aimed at associating types and valences to the physiological responses. After the dictator game and before the beliefs elicitation, we asked participants to report their emotional state when they learned (or were reminded) of the actual wage for the real-effort task. On ten successive screens, we presented to the participants one emotion and they had to report the intensity of this emotion on a 7-point scale, ranging from "no emotion at all" to "high intensity of emotion". The 10 emotions were: anger, surprise, disappointment, joy, elation, jealousy, rage, frustration, irritation and sadness.

\subsubsection{Online questionnaire on risk and loss aversion}

The participants' reaction to falling below or exceeding expected wage might be affected by loss aversion, because participants who want to avoid losses ex-ante might also react more strongly to losses. Therefore, at least one day before the session in the laboratory, participants had to fill out an incentivized online questionnaire aimed at measuring risk and loss aversion according to the method introduced by Eckel and Grossman (2008) (see Appendix B). Participants had to choose one lottery in two consecutive sets of six binary lotteries displayed on their screen. The expected payoff of the lotteries ranged from $€ 2.8$ to $€ 3.6$, and variance increased with the expected payoff. The first set is used to elicit risk attitudes in the gain domain, since expected payoffs are always positive. The second set elicits risk attitudes in the loss domains. In order to keep the expected payoff equal, for the second set of lotteries participants were endowed with $€ 4$. Participants were told that their choice in one of the two sets of lotteries would be chosen randomly for payment, and that they would be informed of the outcome of the lottery at the end of the lab session, when receiving their payment. We classify participants who choose a less risky lottery in the loss domain than in the gain domain as loss-averse. In addition

\footnotetext{
${ }^{4}$ We let participants choose when to reveal the actual wage to minimize the surprise linked to the mere displaying of the actual wage. Surprise affects SCRs and is not of interest in our experiment.
} 
to this two lottery choices, we collected participants' demographics.

We separate these measures from the core of the experiment to avoid cross-contamination between behavior in the core of the experiment and the choices in the risk and loss aversion elicitation.

\subsection{Procedures}

The experiment was conducted at the physio-lab of Gate-lab in Lyon, France. Participants were recruited using hroot (Bock et al., 2014). The online questionnaire was programmed with LimeSurvey (Limesurvey GmbH, 2012) and the laboratory experiment with JAVA. Before the first session, we randomly drew a sequence of conditions thanks to an algorithm that is available upon request. This algorithm ensures that each of the wage levels would be implemented one third of the time. Given this sequence, we planned our sessions by pairs: a Ref and a NoRef condition for the level of wage determined by the algorithm. The use of the algorithm was explained to the participants in the instructions to avoid deception.

In the invitation mailing, participants were informed that we would use physiological measures. We also told them that they would have to fulfill an online questionnaire at least one day before the session. Upon arrival, participants were given an informed consent form detailing the skin conductance measure. In order to participate, they had to explicitly tick the box indicating consent and sign the form. They were informed that they could freely leave the lab if they did not agree, in this case they would receive the amount corresponding to their choice in the online risk aversion task plus $€ 5$. All participants gave their consent.

After consent, an experimentalist took participants to a bathroom where they were invited to wash their hands thoroughly with a special soap to improve the accuracy of the electro-dermal measures. They then drew a sticker from an opaque bag, which indicated the cubicle in which they were invited to seat. An experimentalist installed the electrodes on the index and middle finger of the left-hand of each participant. The temperature was maintained close to 23 degrees Celsius in order to improve the accuracy of the electrodermal measures. An experimentalist then distributed the instructions for the two parts altogether, these instructions were read aloud by the same experimenter and participants could ask questions.

Overall, 280 participants participated in 30 sessions. Among them, $58 \%$ were female and the mean age was 23.29 years (Standard Deviation, S.D. hereafter: 6.73). $85.71 \%$ of the participants were students while $5.71 \%$ were unemployed, $5 \%$ were employed and 3.57 
Table 1: Number of participants by experimental condition

\begin{tabular}{c|c|c|c}
\hline \hline & low wage & medium wage & high wage \\
\hline NoRef & $N=44$ & $N=46$ & $N=44$ \\
\hline Ref & $N=52$ & $N=48$ & $N=46$ \\
\hline \hline
\end{tabular}

$\%$ were classified as having another type of activity. Table 1 summarizes the number of participants in each experimental conditions (more details in Table C.1 in Appendix C). ${ }^{5}$

Sessions were run between June 2018 and April 2019. Our procedure insures that, for any given day in which we planned a session, we would have a NoRef condition for a given wage and the corresponding $R e f$ condition. $^{6}$

Sessions lasted on average 45 minutes. The average payoff is $€ 20.47$ (S.D. 4.9) including a $€ 5$ show-up fee and an average of $€ 3.35$ (S.D. 1.9) for the online questionnaire.

\section{Theoretical framework and hypotheses.}

The classical economic models of social preferences are not well-equipped to understand the effects of expected-wage violation on sharing in our design. For instance, for a given level of wage the size of the surplus to be shared is held constant between the NoRef and the Ref conditions so the dictator can choose from the same set of distributions. As a consequence, outcome-based models of social preferences (e.g. Fehr and Schmidt, 1999; Bolton and Ockenfels, 2000) would predict the same transfers in the NoRef and Ref conditions for a given level of wage. Similarly, the intentions of the recipient and procedural fairness are held constant between the NoRef and the Ref conditions so that intention-based models (e.g. reciprocity, Dufwenberg and Kirchsteiger, 2004; Falk and Fischbacher, 2006) or models focusing on the procedure leading to the final distribution (e.g Cappelen et al., 2007; Saito, 2013) do not offer specific predictions.

In this section, we introduce a model that formalizes how we expect our treatments to affect transfers through expectations. Other models have dealt with social preferences in the loss domain. The Reference Dependent Altruism model of Breitmoser and Tan (2013) and Breitmoser and Tan (2014) assumes that individuals weight the payoff of others less in the loss domain than in the gain domain. The inequity aversion model introduced by Boun My et al. (2018) makes an equivalent assumption by introducing a loss aversion parameter only on the dictators' payoffs. Our model relaxes this assumption and shows

\footnotetext{
${ }^{5}$ We invited 12 participants for each sessions, but due to the no-show, we do not have a perfect balance across conditions.

${ }^{6}$ There is one exception: for one session, the software crashed so that we had to trash the data and plan another session some days later to compensate.
} 
that, even when these weights are equal, loss aversion can make a dictator more selfish to close the gap between his expected and achieved payoffs. We think that this feature of our model is interesting because it provides a potential mechanism of why individuals are more selfish in the loss domain. Another distinctive feature of our model is that the dictator forms references about the amount he would send to the recipient. This implies that a dictator might be disappointed (feel a loss) if he is not able to transfer as much as intended. In addition to intuitive appeal, this reflects the recent advances in the modelling of reference dependent preferences, which posit that, as long as an individual derives utility from a consumption or an activity, he may feel losses if he gets less than expected (Köszegi and Rabin, 2006).

The utility function of the dictator is given by Equation 1 .

$$
\begin{aligned}
u= & m\left(\pi_{i}\right)+\alpha m\left(\pi_{j}\right)+\mu\left(m\left(\pi_{i}\right)-m\left(\overline{\pi_{i}}\right)\right) \\
& +\mu\left(\alpha m\left(\pi_{j}\right)-\alpha m\left(\overline{\pi_{j}}\right)\right)
\end{aligned}
$$

$\pi_{i}$ represents the profit of the dictator and $\pi_{j}$ the profit of the recipient. These profits depend on the wage for the real effort task $W$ and the transfer of the dictator in the dictator game, expressed as a share $1-d$ of the endowment $E$.

$$
\begin{aligned}
& \pi_{i}=W+d E \\
& \pi_{j}=W+(1-d) E
\end{aligned}
$$

$m\left(\pi_{i}\right)+\alpha m\left(\pi_{j}\right)$ is the direct utility function. $m()$ represents $i$ 's valuation of his profit with $m^{\prime}()>0$ and $m^{\prime \prime}()<0 . \alpha m()$ represents $i$ 's valuation of the recipient $j$ 's payoff. $\alpha<1$ captures the fact that $i$ values his own profit more than $j$ 's profit, and can be understood as a coefficient of aversion to advantageous inequality. ${ }^{7}$

$\mu\left(m\left(\pi_{i}\right)-m\left(\overline{\pi_{i}}\right)\right)+\mu\left(\alpha m\left(\pi_{j}\right)-\alpha m\left(\overline{\pi_{j}}\right)\right)$ capture reference-dependence. $\overline{\pi_{i}}$ and $\overline{\pi_{j}}$ represent the references profits. It is worth emphasizing that $\bar{\pi}_{j}$ is the dictator's reference about the recipient's profit. We think that the dictator forms references about the recipient's profit naturally follows from the fact that the dictator derives utility from the recipient's profit. We assume that the dictator forms these references when receiving the instructions by formulating expectations about the wage $(\bar{w})$ and the transfer he would choose for this wage $\left(1-\overline{d_{\bar{w}}}\right) .{ }^{8}$ Gains and losses are defined in terms of the differences in utility derived from the expected and realized values of the profits. In plain words, the dictator encodes

\footnotetext{
${ }^{7}$ Given that both participants earn the same wage, the dictator can always choose a transfer that yields him a higher profit than the recipient so introducing aversion to disadvantageous inequality is unnecessary.

${ }^{8}$ Expectations are frequently used as reference in the literature, see for instance Kahneman and Tversky (1979); Köszegi and Rabin (2006); Abeler et al. (2011); Marzilli Ericson and Fuster (2011); O’Donoghue and Sprenger (2018). In our design, expectations constitute natural references.
} 
the utility he derives from his profit as a loss (a gain) if it is below (above) his expectation. Similarly, the dictator encodes the utility he derives from the recipient's profit as a loss (a gain) if it is below (above) his expectation. ${ }^{9}$ Deviations from the expected utilities are encoded into utility by $\mu()$, a classical gain-loss utility function.

$$
\mu(X)= \begin{cases}X^{\gamma} & \text { if } X \geq 0 \\ -\lambda(-X)^{\beta} & \text { if } X<0\end{cases}
$$

$\lambda \geq 1$ captures the fact that individuals may be more sensitive to losses than to gains. $0<\gamma<1$ and $0<\beta<1$ capture diminishing sensitivity respectively in the gain and in the loss domains. Note that the utility function $u$ so defined is continuous and concave in $\pi_{i}$ for values of $\beta$ close to 1 , a local extremum of this function is thus a global extremum (proofs are presented in Appendix D). ${ }^{10}$

In the NoRef conditions, because there is no uncertainty we assume that the dictator rightfully expects the wage that he actually gets, and transfers the amount he was planning to transfer for the expected wage $\left(1-\overline{d_{\bar{w}}}\right)$. As a consequence he incurs no gains or losses relative to his expectations and he chooses the transfer that maximizes his direct utility function. This transfer satisfies the condition displayed in Equation 2.

$$
m^{\prime}\left(\bar{w}+\overline{d_{\bar{w}}} E\right)=\alpha m^{\prime}\left(\bar{w}+\left(1-\overline{d_{\bar{w}}}\right) E\right)
$$

In the Ref conditions, the wage is ex-ante uncertain and can take three values with equal probability: $W \in\left\{w_{l}, w_{m}, w_{h}\right\}$, with $w_{l}<w_{m}<w_{h}$ and $w-w_{l}=w_{h}-w$. As a consequence, the expected wage can be different from the actual wage. We assume that the dictator expects a wage comprised between the low wage and the high wage: $w_{l}<\bar{w}<w_{h}$. A stronger assumption would be that dictators rationally expect the medium wage $\left(w_{m}\right)$. We avoid this assumption as it is well established that people sometimes form their expectations in a non-rational way, for instance because of probability distortion. Adopting this stronger assumption would not change any of our results. This assumption can also be interpreted at the aggregate level: we assume that at least some participants expect more than the low wage and no participants expect less than the low wage. Symmetrically, we assume that some participants expect less than the high wage, but no participants expect more than the high wage. ${ }^{11}$

Proposition 1. A dictator who receives $w_{l}$ transfers less if the expected $\bar{w}>w_{l}$ than if

\footnotetext{
${ }^{9}$ As noted by Köszegi and Rabin (2006), "the sensation of gain or loss due to a departure from the reference point seems closely related to the consumption value attached to the goods in question", which is why we have gains and losses defined over utilities, both for the dictator's and the recipient's profits.

${ }^{10}$ Both the bounds on the value of $\alpha$ and $\beta$ and the fact that $\beta$ is close to 1 reflects empirical calibrations. See Fox and Poldrack (2014) for an overview of the estimates in the literature.

${ }^{11}$ This would imply some heterogeneity in how individuals form expectations for the same prospect, which has been documented in the literature (see e.g. Baillon et al., 2018).
} 
he expected $w_{l}$. The intuition is the following: because $i$ values his profit more than $j$ 's profit, $i$ is more sensitive to the loss of some of his profit than to the loss of some of $j$ 's profit. As a consequence, $i$ reduces his transfers to $j$ in order to reduce the loss in terms of his profit.

Proof. Consider a dictator who receives $w_{l}$. If he expected this wage, i.e., if $w_{l}=\bar{w}$, he transfers $\left(1-\overline{d_{\bar{w}}}\right) E$ according to the condition in Equation 2. If the dictator expected $\bar{w}>w_{l}$, transferring $\left(1-\overline{d_{\bar{w}}}\right) E$ implies losses in both his and the recipient's profits and does not maximize his utility. As a consequence, the dictator adjusts his transfer downward. To see that, we study the derivative of the utility function of the dictator who expected $\bar{w}>w_{l}$ at the point corresponding to the transfer of the dictator who expected $w_{l}$. This derivative is presented in Equation 3.

$$
\begin{aligned}
u^{\prime} & =E\left[m^{\prime}\left(\bar{w}+\overline{d_{\bar{w}}} E\right)-\alpha m^{\prime}\left(\bar{w}+\left(1-\overline{d_{\bar{w}}}\right) E\right)\right. \\
& +\lambda \beta m^{\prime}\left(\bar{w}+\overline{d_{\bar{w}}} E\right)\left(m\left(\overline{\pi_{i}}\right)-m\left(\bar{w}+\overline{d_{\bar{w}}}\right) E\right)^{\beta-1} \\
& \left.-\lambda \beta \alpha m^{\prime}\left(\bar{w}+\left(1-\overline{d_{\bar{w}}}\right) E\right)\left(\alpha m\left(\bar{w}+\left(1-\overline{d_{\bar{w}}}\right) E\right)-\alpha m\left(\overline{\pi_{j}}\right)\right)^{\beta-1}\right]
\end{aligned}
$$

$m^{\prime}\left(\bar{w}+\overline{d_{\bar{w}}} E\right)-\alpha m^{\prime}\left(\bar{w}+\left(1-\overline{d_{\bar{w}}}\right) E\right)=0$, because the planned transfer maximizes the direct utility function. The sign of the derivative in Equation 3 depends only on the sign of $\lambda \beta m^{\prime}\left(\bar{w}+\overline{d_{\bar{w}}} E\right)\left(m\left(\overline{\pi_{i}}\right)-m\left(\bar{w}+\overline{d_{\bar{w}}}\right) E\right)^{\beta-1}-\lambda \beta \alpha m^{\prime}\left(\bar{w}+\left(1-\overline{d_{\bar{w}}}\right) E\right)(\alpha m(\bar{w}+$ $\left.\left.\left(1-\overline{d_{\bar{w}}}\right) E\right)-\alpha m\left(\overline{\pi_{j}}\right)\right)^{\beta-1}$. Using the fact that $m()$ is concave and that $\alpha<1$, we can show that this expression is positive (Some more details are given in Appendix E). At this point, the dictator can increase his utility by reducing his transfer below (1$\left.\overline{d_{\bar{w}}}\right) E$. As a consequence, for the low wage, negative expectation violation reduces the dictator's transfer. Note that the former expression has a direct interpretation: $\lambda \beta m^{\prime}(\bar{w}+$ $\left.\overline{d_{\bar{w}}} E\right)\left(m\left(\overline{\pi_{i}}\right)-m\left(\bar{w}+\overline{d_{\bar{w}}}\right) E\right)^{\beta-1}$ represents the extra utility of keeping slightly more than $\overline{d_{\bar{w}}} E$, which closes the gap between the profit $i$ expected and the profit $i$ actually gets. $\lambda \beta \alpha m^{\prime}\left(\bar{w}+\left(1-\overline{d_{\bar{w}}}\right) E\right)\left(\alpha m\left(\bar{w}+\left(1-\overline{d_{\bar{w}}}\right) E\right)-\alpha m\left(\overline{\pi_{j}}\right)\right)^{\beta-1}$ represents the utility cost of transferring slightly less than $\left(1-\overline{d_{\overline{w_{l}}}}\right)$, which widens the gap between what $i$ expects the recipient would get and the amount the recipient actually gets. Below the reference point, $i$ prefers closing the gap between what he gets and what he was expecting to get, even if it widens the gap between the recipient's profit and what $i$ expected the recipient would get.

Proposition 2. A dictator who receives $w_{h}$ transfers more if the expected $\bar{w}<w_{h}$ than if he expected $w_{h}$. The intuition is that the combination of the concavity of $m()$ and diminishing sensitivity makes the extra units of profit more valuable when transferred to the recipient rather than kept.

Proof. Consider a dictator who receives $w_{h}$. If he expected this wage, i.e. if $w_{h}=\bar{w}$, he transfers $\left(1-\overline{d_{\bar{w}}}\right) E$ according to the condition in Equation 2. If the dictator expected $\bar{w}<w_{h}$, transferring $\left(1-\overline{d_{\bar{w}}}\right) E$ implies gains in both his and the recipient's profits but 
does not maximize his utility. As a consequence, the dictator adjusts his transfer upward. To see that, we study the derivative of the utility function of the dictator who expected $\bar{w}<w_{h}$ at the point corresponding to the transfer of the dictator who expected $w_{h}$. This derivative is presented in Equation 4.

$$
\begin{aligned}
u^{\prime} & =E\left[m^{\prime}\left(\bar{w}+\overline{d_{\bar{w}}} E\right)-\alpha m^{\prime}\left(\bar{w}+\left(1-\overline{d_{\bar{w}}}\right) E\right)\right. \\
& +\gamma m^{\prime}\left(\bar{w}+\overline{d_{\bar{w}}} E\right)\left(m\left(\bar{w}+\overline{d_{\bar{w}}} E\right)-m\left(\overline{\pi_{i}}\right)\right)^{\gamma-1} \\
& \left.-\alpha \gamma m^{\prime}\left(\bar{w}+\left(1-\overline{d_{\bar{w}}}\right) E\right)\left(\alpha m\left(\bar{w}+\left(1-\overline{d_{\bar{w}}}\right) E\right)-\alpha m\left(\overline{\pi_{j}}\right)\right)^{\gamma-1}\right]
\end{aligned}
$$

The sign of the derivative depicted in Equation 4 is determined by the sign of $\gamma m^{\prime}(\bar{w}+$ $\left.\left.\left.\overline{d_{\bar{w}}} E\right)\right)\left(m\left(\bar{w}+\overline{d_{\bar{w}}} E\right)\right)-m\left(\overline{\pi_{i}}\right)\right)^{\gamma-1}-\alpha \gamma m^{\prime}\left(\bar{w}+\left(1-\overline{d_{\bar{w}}}\right) E\right)\left(\alpha m\left(\bar{w}+\left(1-\overline{d_{\bar{w}}}\right) E\right)-\alpha m\left(\overline{\pi_{j}}\right)\right)^{\gamma-1}$. Using a similar argument than previously, we can show that this expression is negative. At this point, the dictator can increase his utility by increasing his transfer above $\left(1-\overline{d_{\bar{w}}}\right) E$. This means that when $i$ expects a given wage but actually receives a higher wage, $i$ transfers more than if he had expected the high wage.

\subsection{Hypotheses}

Hypothesis 1: For the medium wage, participants transfer the same amount in the $R e f_{m e d}$ and NoRe $f_{\text {med }}$ condition. This hypothesis does not follow directly from the model, but hinges on the supplementary hypothesis that participants in the Ref conditions expect to receive the medium wage $w_{m}$.

The previous theoretical framework leads to the following hypotheses:

Hypothesis 2: For the low wage, participants in the $R e f_{\text {low }}$ condition transfer less than participants in the NoRe $f_{\text {low }}$. This follows from proposition 1.

Hypothesis 3: For the high wage, participants in the $R e f_{\text {high }}$ condition transfer more than participants in the NoRe $f_{\text {high }}$ condition. This follows from proposition 2 .

\section{Results}

The average transfer in the dictator game is $€ 1.41$ (S.D. 1.39). This represents $28 \%$ of the $€ 5$ endowment, which is well in line with the literature. ${ }^{12}$ Table 2 reports the average transfer of participants across conditions and the proportion of null transfers.

Interestingly, there is no significant difference in transfers across the different wages in the NoRef conditions, suggesting that there is no wealth effect (two-sided Kruskal-Wallis

\footnotetext{
${ }^{12}$ In a meta-analysis including more than 130 papers and 600 treatments, Engel (2011) finds that dictators give on average $28 \%$ of their endowment.
} 
Table 2: Transfers across conditions

\begin{tabular}{|c|c|c|c|}
\hline & Wage $=5$ & Wage $=10$ & Wage $=15$ \\
\hline \multicolumn{4}{|c|}{ Mean transfers. } \\
\hline NoRef & $1.61(1.41)$ & $1.38(1.39)$ & $1.46(1.45)$ \\
\hline $\operatorname{Ref}$ & $1.21(1.39)$ & $1.18(1.46)$ & $1.68(1.25)$ \\
\hline $\mathrm{p}$-value & 0.114 & 0.505 & 0.345 \\
\hline Overall & $1.39(1.40)$ & $1.28(1.42)$ & $1.57(1.35)$ \\
\hline \multicolumn{4}{|c|}{ Prop. transfer $=0$. } \\
\hline NoRef & 0.27 & 0.41 & 0.36 \\
\hline Ref & 0.46 & 0.46 & 0.24 \\
\hline $\mathrm{p}$-value & 0.062 & 0.683 & 0.225 \\
\hline Overall & 0.38 & 0.44 & 0.30 \\
\hline Notes: & Standard & viation & $\begin{array}{l}\text { parentheses. } \\
\text { hitney tests }\end{array}$ \\
\hline
\end{tabular}

tests, $p=0.697)$. In contrast, transfers in the Ref conditions differ across the different wages (two-sided Kruskal-Wallis tests, $p=0.046$ ). Departures from expected wage seem to have a greater effect on transfers that the actual level of wage.

The comparison of average transfers between Ref and NoRef for a given wage qualitatively match our hypotheses, but the differences do not reach statistical significance at the $5 \%$ level in univariate, non-parametric tests (Two-sided Mann-Whitney tests: Low wage, $p=0.114$; Medium wage $p=0.505$; High wage $p=0.345$ ).

We ran regressions explaining transfers, pooling all the data from the experiment. We interact a dummy variable indicating the Ref conditions, with a categorical variable indicating the wage level. In Table 3, we report the marginal treatment effects for the different levels of wage. Models (1)-(3) are Tobit models (37\% of transfers censored at 0). Models (4)-(6) are Logit models, explaining the decision to transfer zero. Models (2)-(3) and (5)-(6) control for individual characteristics, namely age, gender and dummies for whether the participant is a student, whether he or she is loss-averse and whether he or she has already participated in economic experiments. ${ }^{13}$ Models (3) and (6) include a categorical variable indicating the month in which the data was collected. ${ }^{14}$ Robust standard errors are clustered at the session level. ${ }^{15}$

\footnotetext{
${ }^{13}$ Participants who chose a lottery with less variance in the loss domain than in the gain domain in the online questionnaire are categorized as loss averse.

${ }^{14}$ We ran sessions in early summer which corresponds to the end of the academic year, in early fall which corresponds to the beginning of the academic year and in early spring the following year. The participants differ on observable and might differ on non-observable characteristics (see Table C.1 in Appendix C).

${ }^{15}$ There are several reasons to cluster standard errors at the session level even in one-shot experiments (see e.g. Fréchette, 2012). For instance, the experimental procedure to set-up the physiological measures
} 
Table 3: Treatment effects over the different levels of wage.

\begin{tabular}{lcccccc}
\hline \hline & $\begin{array}{c}(1) \\
\text { Transfer }\end{array}$ & $\begin{array}{c}(2) \\
\text { Transfer }\end{array}$ & $\begin{array}{c}(3) \\
\text { Transfer }\end{array}$ & $\begin{array}{c}(4) \\
\text { Transfer=0 }\end{array}$ & $\begin{array}{c}(5) \\
\text { Transfer=0 }\end{array}$ & $\begin{array}{c}(6) \\
\text { Transfer=0 }\end{array}$ \\
\hline Treatment effect & & & & & & \\
Wage=5 & $-0.731^{* *}$ & $-0.610^{*}$ & $-0.520^{* *}$ & $0.189^{* *}$ & $0.167^{*}$ & $0.155^{* *}$ \\
& $(0.305)$ & $(0.322)$ & $(0.255)$ & $(0.093)$ & $(0.091)$ & $(0.063)$ \\
Wage=10 & -0.301 & -0.380 & -0.279 & 0.045 & 0.0604 & 0.018 \\
& $(0.319)$ & $(0.294)$ & $(0.322)$ & $(0.066)$ & $(0.062)$ & $(0.058)$ \\
Wage=15 & 0.406 & 0.491 & $0.498^{*}$ & $-0.125^{*}$ & $-0.145^{* *}$ & $-0.150^{* *}$ \\
& $(0.290)$ & $(0.300)$ & $(0.280)$ & $(0.074)$ & $(0.068)$ & $(0.069)$ \\
\hline Individual Char. & No & Yes & Yes & No & Yes & Yes \\
Month F.E. & No & No & Yes & No & No & Yes \\
\hline Observations & 280 & 280 & 280 & 280 & 280 & 280 \\
\hline \hline
\end{tabular}

Notes: Robust standard errors clustered at the session level are in parentheses. (1)-(3) are Tobit models. (4)-(6) are Logit models. Marginal effects reported. Treatment effect corresponds to the marginal effect of the Ref condition. ${ }^{*} p<0.10,{ }^{* *} p<0.05,{ }^{* * *} p<0.01,{ }^{* * * *} p<0.001$.

The outcome of these regressions supports the following results:

Result 1: For the $€ 10$ wage, the $\operatorname{Ref}$ condition has no significant impact on transfers, neither at the intensive nor at the extensive margin. This result supports Hypothesis 1 . While negative, none of the parameters capturing treatment effects for the $€ 10$ wage are significant in the regressions.

Result 2: For the $€ 5$ wage, the Ref condition reduces transfers significantly, both at the intensive and extensive margins. The estimates presented in Table 3 show that participants in the Reflow condition transfer between $€ 0.731$ and $€ 0.52$ less on average than participants in the NoReflow condition (Table 3, Model 1: $p=0.016$, Model 2: $p=0.058$, Model 3: $p=0.041)$. In addition, we estimate that participants in the $R e f_{\text {low }}$ are between 18.9 and 15.5 percentage points more likely to transfer zero than those in the NoRe $f_{\text {low }}$ condition depending on the specification (Table 3, Model 4: $p=0.044$, Model 5: $p=0.070$, Model 6: $p=0.015)$.

As $€ 5$ is the lowest possible wage, it is probably encoded as a loss by participants. Lossaverse participants in the $R e f_{\text {low }}$ condition transfer $€ 0.68(\mathrm{n}=19$, S.D. $=0.99)$ on average while non loss-averse transfer $€ 1.5(\mathrm{n}=33$, S.D. $=1.5$, Two-sided Mann-Whitney test: $p=0.042)$. Out of the 19 loss-averse participants in the Reflow condition, 12 (63\%) transferred zero, against 12 out of the 33 non loss-averse participants (36\%, two-sided implied interactions between participants and with the experimenter that are hardly measurable and made the room composition in terms of gender salient which can impact behavior (Castillo et al., 2019). 
Fischer exact test: $p=0.086$ ). The results of these tests are validated by regression analysis in which we contrast the treatment effects on loss-averse and on non loss-averse participants. These results are reported in Table F.1 in Appendix F. This suggests that the treatment impacts loss-averse participants more than it affects non loss-averse ones.

Result 3: For the $€ 15$ wage, the $R e f$ condition increases transfers at the extensive margin. The estimated treatment effects for the $€ 15$ wage are between $€ 0.4$ and $€ 0.5$ depending on the specification, but only one is marginally significant (Table 3 , Model 3,p=0.076). On the other hand, we estimate that participants in the $R e f_{\text {high }}$ condition are between 12.5 and 15.0 percentage points less likely to transfer zero than participants in the NoRe $f_{\text {high }}$ condition (Table 3, Model 4: $p=0.097$, Model 5: $p=0.036$, Model 6, $p=0.032)$.

Loss-averse participants in the $R e f_{\text {high }}$ condition transfer $€ 2.11(\mathrm{n}=13$, S.D. $=0.98)$ on average while non loss-averse transfer $€ 1.50$ ( $\mathrm{n}=33$, S.D. $=1.31$, two-sided MannWhitney test: $p=0.066)$. Out of the 13 loss-averse participants in the $R e f_{\text {high }}$ condition, only 1 transferred zero, against 10 out of the 33 non-loss-averse participants (30\%, twosided Fischer exact test: $p=0.141$ ). These results are validated by the regression analysis reported in Table F.1 in Appendix F. Loss aversion seems to mediate the effect of earning a wage higher than expected on transfers.

In the next paragraphs, we discuss the role of emotions as a channel for the previous results. ISCRs are always greater in the Ref than in the NoRef conditions (two-sided Mann-Whitney tests: $p$-values are lower than 0.001 for every levels of wage, see Figure H.1 in Appendix H). This shows that the revelation of the actual wage to participants who were uncertain triggers emotional responses. Yet, emotional responses are not different across the levels of wage in both the Ref conditions and the NoRef conditions (two-sided Kruskal-Wallis test: Ref, $p=0.624 ;$ NoRef, $p=0.116$ ). In the NoRef conditions, participants already knew their wage level, this explains why there is no difference between the ISCRs of the different wage level. In the Ref conditions, this lack of difference is certainly explained by anticipatory emotions which are independent from the actual level of wage. Comparing the anticipatory ISCRs with the ISCRs at the time of the wage revelation, we find that the former is significantly larger than the latter but only in Reflow and Ref $f_{\text {med }}$ (Signed-rank tests: Reflow and $R e f_{\text {med }}, p-$ value $<0.001$; $R e f_{\text {high }}, p-v a l u e=0.755$. See Appendix H for details on the analysis.).

As stated earlier, we asked participants to report the intensity of their emotions to associate a valence to ISCRs data. We separate the items of the questionnaire in "positive" emotions (Joy and elation) and "negative" emotions (Anger, disappointment, rage, irritation, jealousy, frustration, sadness). For each individual, we average the responses to 
"positive" items and to "negative" items to compute individual indexes of "positive" and "negative" emotions. ${ }^{16}$

Table 4 reports the average of positive and negative emotions indexes across wage level and treatment. Participants report more negative emotions and less positive emotions in the Ref than in the NoRef condition for the $€ 5$ and $€ 10$ wage, while the opposite is found for the $€ 15$ wage.

Table 4: Average positive and negative emotion indexes.

\begin{tabular}{|c|c|c|c|}
\hline & Wage $=5$ & Wage $=10$ & Wage $=15$ \\
\hline \multicolumn{4}{|c|}{ Positive emotion. } \\
\hline NoRef & $3.01(1.48)$ & $3.65(1.30)$ & $3.87(1.44)$ \\
\hline $\operatorname{Ref}$ & $1.40(0.76)$ & $2.65(1.24)$ & $4.83(1.33)$ \\
\hline $\mathrm{p}$-value & $<0.001$ & $<0.001$ & 0.001 \\
\hline \multicolumn{4}{|c|}{ Negative emotion. } \\
\hline NoRef & $1.39(0.62)$ & $1.19(0.34)$ & $1.37(0.78)$ \\
\hline Ref & $3.22(1.37)$ & $2.44(1.26)$ & $1.38(0.99)$ \\
\hline p-value & $<0.001$ & $<0.001$ & 0.052 \\
\hline
\end{tabular}

Notes: Standard deviation are in parentheses. $p$-values for two-sided Mann-Whitney tests.

To further analyze the role of emotions, we separate participants according to the strength of their emotional response around the revelation of their actual wage. This is done by splitting participants according to the median ISCR in each condition separately. For instance, a participant in the Re $f_{\text {low }}$ condition is classified as having high emotional reaction if his ISCR is greater than the median ISCR of participants in the $R e f_{\text {low }}$ condition. We ran regressions explaining transfers by the interaction between wage and a dummy variable indicating a high emotional reaction on the data from the $R e f$ conditions. The marginal effect of being in the high ISCR group for each level of wage is reported in Table 5. The results show that participants whose ISCRs are above the median transfer less in the $R e f_{\text {low }}$ condition than those whose ISCRs are below. This suggests that participants who experience more emotions upon a loss tend to transfer less in reaction to a loss. In contrast, participants with high emotional responses do not transfer different amounts than others in the Re $f_{\text {high }}$ condition.

We have shown that high ISCRs predict treatment effects and that ISCRs are mostly explained by anticipatory emotions. We now explore whether ISCRs specifically resulting from anticipatory emotions are predictive of our treatment effects. To do that, we run

\footnotetext{
${ }^{16}$ The questionnaire has a good reliability overall (Cronbach's alpha=0.84). Table G.1 in Appendix G gives more details about the structure of correlations between the items from the questionnaire.
} 
Table 5: The effect of "high emotional arousal" over the different levels of wage for the Ref conditions.

\begin{tabular}{lcccccc}
\hline \hline & $(1)$ & $(2)$ & $(3)$ & $(4)$ & $(5)$ & $(6)$ \\
& Transfer & Transfer & Transfer & Transfer=0 & Transfer=0 & Transfer=0 \\
\hline Effect of high emotional arousal at: & & & & & & \\
Wage=5 & $-1.971^{* *}$ & $-2.117^{* *}$ & $-2.162^{* *}$ & $0.385^{* *}$ & $0.424^{* *}$ & $0.426^{* *}$ \\
& $(0.947)$ & $(1.053)$ & $(1.047)$ & $(0.182)$ & $(0.189)$ & $(0.173)$ \\
Wage $=10$ & -0.407 & -0.367 & -0.346 & $>0.001$ & 0.005 & -0.016 \\
& $(0.725)$ & $(0.677)$ & $(0.711)$ & $(0.183)$ & $(0.179)$ & $(0.185)$ \\
Wage=15 & -0.233 & -0.239 & -0.247 & -0.0435 & -0.0298 & -0.0157 \\
& $(0.426)$ & $(0.345)$ & $(0.322)$ & $(0.075)$ & $(0.058)$ & $(0.062)$ \\
\hline Individual Char. & No & Yes & Yes & No & Yes & Yes \\
Month F.E. & No & No & Yes & No & No & Yes \\
\hline Observations & 146 & 146 & 146 & 146 & 146 & 146 \\
\hline \hline
\end{tabular}

Notes: Robust standard errors clustered at the session level are in parentheses. (1)-(3) are Tobit models. (4)-(6) are Logit models. Marginal effects reported. High emotional arousal corresponds to the marginal effect of having a ISCRs higher than the median ISCRS in a given condition. ${ }^{*} p<0.10,{ }^{* *} p<0.05,{ }^{* * *} p<0.01$.

the same regressions than the ones reported in Table 5 but focusing alternatively on the ISCRs in the anticipation phase and on the ISCRs in the revelation phase (see Table H.2 and Table H.3 in Appendix H). These regressions show that the predictive power of ISCR is mostly due to the ISCRs recorded before the revelation of the wage. This emphasizes the role of anticipatory emotions in our treatment effects. Furthermore, participants who have a higher than median anticipatory ISCRs are significantly more likely to be loss averse (Two-sided Fischer's exact test, $p=0.011) .{ }^{17}$ Altogether, this suggests the existence of a link between our treatment effect, loss aversion and anticipatory emotions.

\section{Conclusion}

In this paper, we tested whether negative and positive violations of expected wage for a real effort task affect giving in a subsequent dictator game. We found some support for this hypothesis. In particular, participants who receive the low wage transferred less when they were informed of the existence of higher potential levels of wage than when they were not informed. The results are more nuanced when participants receive the high wage, as in this case being informed of the other level of wage only decreases the likelihood of giving zero.

The effects are found by manipulating participants' expected wage, holding the actual wage constant. As a consequence, wealth effects do not confound our results. By design, we also mute reciprocity concerns since the recipients in the dictator game cannot be held accountable for the actual wage in the real effort task. Our interpretation is that

\footnotetext{
${ }^{17}$ As a robustness check we made a Pearson's correlation test between the score of loss aversion and the anticipatory ISCRs and we found a significant correlation at the $10 \%$ level $(p=0.052)$.
} 
earning a wage that is below expectations is encoded as a loss and dictators reduce their transfers in order to compensate for this loss. Earning a wage exceeding expectations may symmetrically be encoded as a gain, but since our results are weaker in this case, a possibility is that in our setting losses may have greater behavioral consequences than gains. In addition, we found greater behavioral effects on loss averse participants. This comforts our analysis in terms of reference dependence: it makes intuitive sense that the more loss averse individuals are more willing to try to close the gap between what they expected to be paid and what they actually get paid. In addition, this is consistent with classical results showing that loss aversion mediates the effect of prior losses on subsequent behavior (e.g. Thaler and Johnson, 1990).

Our analysis leaves expectations as a (almost) free parameter, because our theoretical model and our interpretation only assume that participants expect a wage comprised between the low and the high wage (or that at least some participants would consider the low wage as a loss and the high wage as a gain). While not significant, we observe a tendency toward a negative treatment effect for the medium wage $(€ 10)$. Emotion data show that participants felt more negative emotions in the $R e f_{\text {med }}$ than in the NoRe $f_{\text {med }}$ condition. It reinforces the idea that participants do not uniquely "rationally" expect the medium wage and suggests that some participants consider any wage below $€ 15$ as a loss. This is an alternative interpretation of the asymmetry between the treatment effects for the low and the high wage. It would be interesting to dig into the expectations of participants, and especially the individual heterogeneity and its underpinnings.

In our experiment, the loss (or gains) is experienced in the first part of the experiment, before the decision to share. In the second part of the experiment, we gave dictators a separate endowment, independent of whether they experienced a gain or a loss in the previous part. This might have favored bracketing by participants: they might have considered the two parts of the experiment separately. Despite this arguably conservative design choice, we still find an effect, suggesting that participants did not bracket to the full extent. This is interesting in itself, but also reinforces the idea that experimenters should be very careful to the potential spillovers from one part of the game to another. ${ }^{18}$

The analysis of physiological and self-reported emotional data shows that our treatments trigger emotional reactions, suggesting that emotions are a channel of the effects identified in this paper. By formally showing that falling below or exceeding one's reference point triggers emotional responses, our study reinforces the idea that the behavioral effects of losses (and gains) have visceral drives (Card and Dahl, 2011; Andrade and Ariely,

\footnotetext{
${ }^{18}$ Of course, we are not the first to note that. For instance, Chowdhury and Jeon (2014) show that changing the show-up fee participants receive at the beginning of an experiment impacts transfers in a dictator game later in the experiment.
} 
2009). More generally, it also contributes to the literature exploring the role of emotions in economic behavior (e.g., Loewenstein, 2000; Loewenstein and Lerner, 2003; Rick and Loewenstein, 2008; Battigalli et al., 2019). Unlike previous research which directly manipulates mood or emotional state and measure its effect on other regarding preferences, (e.g., Capra, 2004; Kirchsteiger et al., 2006; Tan and Forgas, 2010; Ibanez et al., 2017; Pérez-Dueñas et al., 2018), we investigated how emotions can explain the effect of the violations of expectations on a subsequent pro-social behavior. Our paper provides suggestive evidence that anticipatory emotions are a better predictor of the effect of losses and gains on transfers than emotions after revelation. Furthermore, anticipatory emotional reaction seems related to the participants' degree of loss-aversion, in line with previous research (e.g., Knutson and Greer, 2008; Corgnet et al., 2020). Overall, our results show that the role of emotions on reference dependent preference is yet to be understood. Some research on how, when and why exactly anticipatory emotions are good predictors of the effects of gains and losses on behavior may be in order.

Our results contribute to the literature studying social preferences, and more specifically how the context of the decision impacts giving (e.g., List, 2007; Bardsley, 2008). Our manipulation allows to observe the transfers of dictators whose payoffs differ from their reference, and the data point to the existence of a trade-off between social preferences and the natural tendency of loss averse people to chase losses: losses relative to their reference payoff reduce the concern for other while gains slightly increase it. Self-commitment to act altruistically seems to yield in front of the dis-utility linked to incurring private losses. This adds to the literature studying the role of reference in other regarding preference (e.g., Thunstrom, 2019; Boun My et al., 2018; Cochard et al., 2020; Fiedler and Hillenbrand, 2020).

Our results also speak to the literature studying the effects of wage cuts on social preferences (e.g., Kube et al., 2012; Ockenfels et al., 2015). In contrast with previous contributions, in our experiment, we mute inequality concerns, as all the participants in a given session earn the same wage ; and reciprocity, as the recipient is not accountable for the actual level of wage. As a consequence, we provide evidence that workers may compare their wages to the wages they could have received in a different state of the world, independently of social comparison or wage dynamic. Moreover, losses and gains in terms of wage affect social preferences, rather than effort provision. This means that how workers evaluate their wage relative to a reference might impact the social capital of firms, and promote or impede cooperation between workers. 


\section{References}

Abeler, J., A. Falk, L. Goette, and D. Huffman (2011). Reference Points and Effort Provision. American Economic Review 101(April), 470-492.

Andrade, E. B. and D. Ariely (2009). The enduring impact of transient emotions on decision making. Organizational Behavior and Human Decision Processes 109(1), 1-8.

Antinyan, A. (2014). Loss and other-regarding preferences. Department of Management, Università Ca'Foscari Venezia Working Paper 3.

Baillon, A., H. Bleichrodt, and V. Spin (2018). Searching for the reference point. Management Science.

Bardsley, N. (2008). Dictator game giving: altruism or artefact? Experimental Economics 11(2), 122-133.

Battigalli, P., M. Dufwenberg, and A. Smith (2019). Frustration, aggression, and anger in leader-follower games. Games and Economic Behavior 117, 15 - 39.

Benedek, M. and C. Kaernbach (2010). A continuous measure of phasic electrodermal activity. Journal of Neuroscience Methods 190(1), 80-91.

Bock, O., I. Baetge, and A. Nicklisch (2014). hroot: Hamburg Registration and Organization Online Tool. European Economic Review 71(C), 117-120.

Bolton, G. E. and A. Ockenfels (2000). Erc: A theory of equity, reciprocity, and competition. The American Economic Review 90(1), 166-193.

Bosman, R. and F. van Winden (2002). Emotional Hazard in a Power-to-Take Experiment. The Economic Journal 112(476), 147-169.

Boun My, K., N. Lampach, M. Lefebvre, and J. Magnani (2018). Effects of gain-loss frames on advantageous inequality aversion. Journal of the Economic Science Association 4(2), 99-109.

Bradley, M. M. and P. J. Lang (2000). Measuring emotion: Behavior, feeling, and physiology. In Cognitive neuroscience of emotion., Series in affective science., pp. 242-276. New York, NY, US: Oxford University Press.

Breitmoser, Y. and J. H. Tan (2013). Reference dependent altruism in demand bargaining. Journal of Economic Behavior and Organization 92, 127-140.

Breitmoser, Y. and J. H. Tan (2014). Reference dependent altruism.

Buchan, N., R. Croson, E. Johnson, and G. Wu (2005). Gain and loss ultimatums. Advances in Applied Microeconomics 13, 1-23. 
Buffat, J. (2016). Charitable preferences and expectation-based reference dependence: An experiment on charitable giving.

Cappelen, A. W., A. D. Hole, E. Ø. Sørensen, and B. Tungodden (2007). The pluralism of fairness ideals: An experimental approach. American Economic Review 97(3), 818827.

Capra, C. M. (2004). Mood-driven behavior in strategic interactions. The American Economic Review 94(2), 367-372.

Card, D. and G. B. Dahl (2011). Family violence and football: The effect of unexpected emotional cues on violent behavior. Quarterly Journal of Economics 126(1), 103-143.

Castillo, M., G. Leo, and R. Petrie (2019). Room composition effects on risk taking by gender. Experimental Economics.

Chowdhury, S. M. and J. Y. Jeon (2014). Impure altruism or inequality aversion?: An experimental investigation based on income effects. Journal of Public Economics 118, $143-150$

Cochard, F., A. Flage, G. Grolleay, and A. Sutan (2020). Are individuals more generous in loss contexts? Social Choice and Welfare.

Corgnet, B., C. Cornand, and N. Hanaki (2020). Tail events, emotions and risk taking. working paper or preprint.

Dana, J., R. A. Weber, and J. X. Kuang (2007). Exploiting moral wiggle room: experiments demonstrating an illusory preference for fairness. Economic Theory 33(1), $67-80$.

Drouvelis, M. and B. Grosskopf (2016). The effects of induced emotions on pro-social behaviour. Journal of Public Economics 134, 1-8.

Dufwenberg, M. and G. Kirchsteiger (2004). A theory of sequential reciprocity. Games and Economic Behavior 47(2), 268-298.

Eckel, C. C. and P. J. Grossman (2008). Forecasting risk attitudes: An experimental study using actual and forecast gamble choices. Journal of Economic Behavior \& Organization 68(1), 1 - 17 .

Engel, C. (2011). Dictator games: A meta study. Experimental Economics 14(4), 583610.

Falk, A. and U. Fischbacher (2006). A theory of reciprocity. Games and Economic Behavior 54, 293-315. 
Fehr, E. and K. M. Schmidt (1999). A theory of fairness, competition, and cooperation. The Quarterly Journal of Economics 114(3), 817-868.

Fiedler, S. and A. Hillenbrand (2020). Gain-Loss Framing in Interdependent Choice. Games and Economic Behavior.

Fox, C. R. and R. A. Poldrack (2014). Appendix: Prospect theory and the brain. In P. W. Glimcher and E. Fehr (Eds.), Neuroeconomics (Second Edition) (Second Edition ed.)., pp. 533 - 567. San Diego: Academic Press.

Fréchette, G. R. (2012). Session-effects in the laboratory. Experimental Economics 15(3), 485-498.

Garbarino, E., R. Slonim, and M. C. Villeval (2019). Loss aversion and lying behavior. Journal of Economic Behavior \& Organization 158, 379 - 393.

Heidhues, P. and B. Köszegi (2008). Competition and price variation when consumers are loss averse. American Economic Review 98(4), 1245-68.

Ibanez, L., N. Moureau, and S. Roussel (2017). How do incidental emotions impact proenvironmental behavior? Evidence from the dictator game. Journal of Behavioral and Experimental Economics 66, 150-155.

Kahneman, D. and A. Tversky (1979). Prospect theory: An analysis of decision under risk. Econometrica $47(2), 263-291$.

Kirchsteiger, G., L. Rigotti, and A. Rustichini (2006). Your morals might be your moods. Journal of Economic Behavior and Organization 59(2), 155-172.

Knutson, B. and S. M. Greer (2008). Anticipatory affect: Neural correlates and consequences for choice. Philosophical Transactions of the Royal Society B: Biological Sciences 363(1511), 3771-3786.

Köszegi, B. and M. Rabin (2006). A model of reference-dependent preferences. The Quarterly Journal of Economics 121(4), 1133-1165.

Kube, S., M. A. Maréchal, and C. Puppe (2012). Do wage cuts damage work morale? evidence from a natural field experiment. Journal of the European Economic Association 11(4), 853-870.

Lerner, J. S., Y. Li, P. Valdesolo, and K. S. Kassam (2015). Emotion and Decision Making. Annual Review of Psychology 66(1), 799-823.

Limesurvey GmbH (2012). LimeSurvey: An Open Source survey tool. Hamburg, Germany: LimeSurvey Project. 
List, J. A. (2007). On the Interpretation of Giving in Dictator Games. Journal of Political Economy 115(3), 482-493.

Loewenstein, G. (2000). Emotions in economic theory and economic behavior. American Economic Review 90(2), 426-432.

Loewenstein, G. and J. Lerner (2003). The role of affect in decision making. In R. Davidson, H. Goldsmith, and K. Scherer (Eds.), Handbook of Affective Science, pp. 619-642. Oxford: Oxford University Press.

Marzilli Ericson, K. M. and A. Fuster (2011). Expectations as Endowments: Evidence on Reference-Dependent Preferences from Exchange and Valuation Experiments. The Quarterly Journal of Economics 126(4), 1879-1907.

Matarazzo, O., B. Pizzini, and C. Greco (2020). Influences of a Luck Game on Offers in Ultimatum and Dictator Games: Is There a Mediation of Emotions? Frontiers in Psychology 11 (February), 1-15.

Neumann, T., S. Kierspel, I. Windrich, R. Berger, and B. Vogt (2018). How to split gains and losses? experimental evidence of dictator and ultimatum games. Games 9(4).

Neumann, T., S. Schosser, and B. Vogt (2017). Ultimatum bargaining over losses and gains - an experimental comparison. Social Science Research 67, 49 - 58.

Ockenfels, A., D. Sliwka, and P. Werner (2015). Bonus payments and reference point violations. Management Science 61(7), 1496-1513.

O'Donoghue, T. and C. Sprenger (2018). Chapter 1 - reference-dependent preferences. In B. D. Bernheim, S. DellaVigna, and D. Laibson (Eds.), Handbook of Behavioral Economics - Foundations and Applications 1, pp. 1-77. North-Holland.

Pérez-Dueñas, C., M. F. Rivas, O. A. Oyediran, and F. García-Torres (2018). Induced negative mood increases dictator game giving. Frontiers in Psychology 9, 1-4.

Persson, E. (2018). Testing the impact of frustration and anger when responsibility is low. Journal of Economic Behavior \& Organization 145, 435 - 448.

Rabin, M. (1993). Incorporating fairness into game theory and economics. The American Economic Review 83(5), 1281-1302.

Rick, S. and G. Loewenstein (2008). The role of emotion in economic behavior. In M. Lewis, J. M. Haviland-Jones, and L. B. Feldman (Eds.), Handbook of emotions., 3rd ed., pp. 138-156. The Guilford Press.

Ring, P. and U. Schmidt (2019). Skin conductance responses in anticipation of gains and losses. Journal of the Economic Science Association 5(1), 38-50. 
Saito, K. (2013). Social preferences under risk: Equality of opportunity versus equality of outcome. American Economic Review 103(7), 3084-3101.

Sokol-Hessner, P., M. Hsu, N. G. Curley, M. R. Delgado, C. F. Camerer, and E. A. Phelps (2009). Thinking like a trader selectively reduces individuals' loss aversion. Proceedings of the National Academy of Sciences of the United States of America 106(13), 50355040 .

Tan, H. B. and J. P. Forgas (2010). When happiness makes us selfish, but sadness makes us fair: Affective influences on interpersonal strategies in the dictator game. Journal of Experimental Social Psychology 46(3), 571-576.

Thaler, R. H. and E. J. Johnson (1990). Gambling with the house money and trying to break even: The effects of prior outcomes on risky choice. Management science 36(6), 643-660.

Thunstrom, L. (2019). Preferences for fairness over losses. Journal of Behavioral and Experimental Economics 83, 101469.

Tversky, A. and D. Kahneman (1991). Loss aversion in riskless choice: A referencedependent model. The Quarterly Journal of Economics 106(4), 1039-1061. 


\section{A Instructions}

Thank you for your participation in this experiment on decision making. You are not allowed to communicate with other participants for the length of the experimental session. Your decisions are anonymous. In addition, because we record your skin conductance, please avoid making noise throughout the session.

The session is composed of three parts. The instructions hereafter describe the content of Parts 1 and 2. The instructions for the third part will be displayed on your screen.

Your decisions can make you earn money. Your payoffs, except for Part 1, will be payed in cash, in private at the end of the session and in a separate room.

We will read the instructions together. Should you have any question, raise your hand. An experimentalist will come to you.

\section{Part 1}

For this part, you have to perform 120 encoding tasks. The task consists in encoding a letter from the alphabet into a number. To know the number corresponding to the letter, you must use a correspondence table that is displayed on your screen. After inputting the letter in the dedicated field, you must validate your answer. If your answer is correct, you go on to the next letter; otherwise you have to input a new number. For each letter, the correspondence table changes.

\section{[The next paragraph depends on the conditions.]}

[NoRef condition (wage $=€ 5$ ).]

For this task, you will receive a payoff. An envelop containing $€ 5$ was placed on your desk for this purpose. The content of the envelop of every participants in the session is the same. The content of the envelop is your payoff for the first part. The content of the envelop will be reminded to you at the end of Part 1.

[NoRef condition (wage $=€ 10)$.

For this task, you will receive a payoff. An envelop containing $€ 10$ was placed on your desk for this purpose. The content of the envelop of every participants in the session is the same. The content of the envelop is your payoff for the first part. The content of the envelop will be reminded to you at the end of Part 1. 
[NoRef condition (wage $=€ 15)$.

For this task, you will receive a payoff. An envelop containing $€ 15$ was placed on your desk for this purpose. The content of the envelop of every participants in the session is the same. The content of the envelop is your payoff for the first part. The content of the envelop will be reminded to you at the end of Part 1.

[Ref conditions.] ***

For this task, you will receive a payoff. An envelop containing $€ 10$ was placed on your desk for this purpose. Nevertheless, at the end of the encoding tasks, a random draw will determine your definitive payoff for this part. This random draw was done at the beginning of the set up of the experiment, before the first session, for every sessions. There is 1 chance out of 3 that the content of the envelop remains unchanged, 1 chance out of 3 that we withdraw $€ 5$ from the envelop and 1 chance out of 3 that we add $€ 5$ in the envelop.

The random draw is at the session level, so that the content of the envelop of every participant in a given session is the same. The random draw does not depend on your behavior or on the behavior of the other participants. The final content of the envelop is your payoff for Part 1.

$* * *$

\section{Part 2}

In this part, the decisions involve a person $\mathrm{A}$ and a person $\mathrm{B}$. Person $\mathrm{A}$ receives a $€ 5$ endowment. She decides how much she wants to transfer to Person B. Every amount between 0 and 5 are possible, rounded to 10 cents. Person A keeps the amount she does not transfer.

Person B does not receive an endowment. She earns the amount that Person A transferred. She has no decision to make.

Every participants in the session take the decision as Person A. At the end of the session, the software will randomly match participants of the session in pairs. For each pair, the software will randomly select a Person A and a Person B. For Person A, her decision will determine her payoff for this part. For Person B, the decision of the Person A to whom 
she is matched will determine her payoff for this part.

Payoff for Part 2 will be payed at the end of the session. 


\section{B Screenshots}

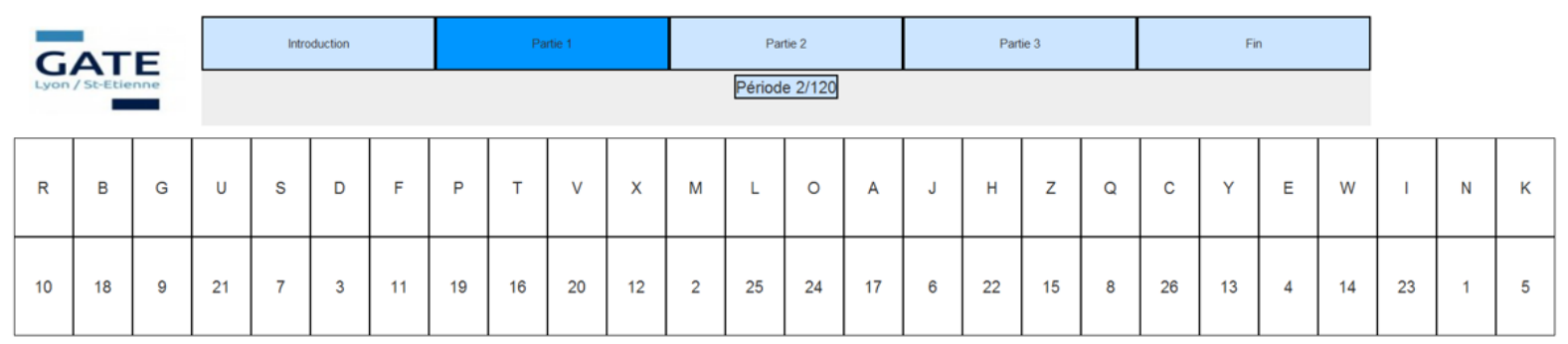

Vous devez encoder la lettre: $F$

Figure B.1: Screenshot of the real effort task.

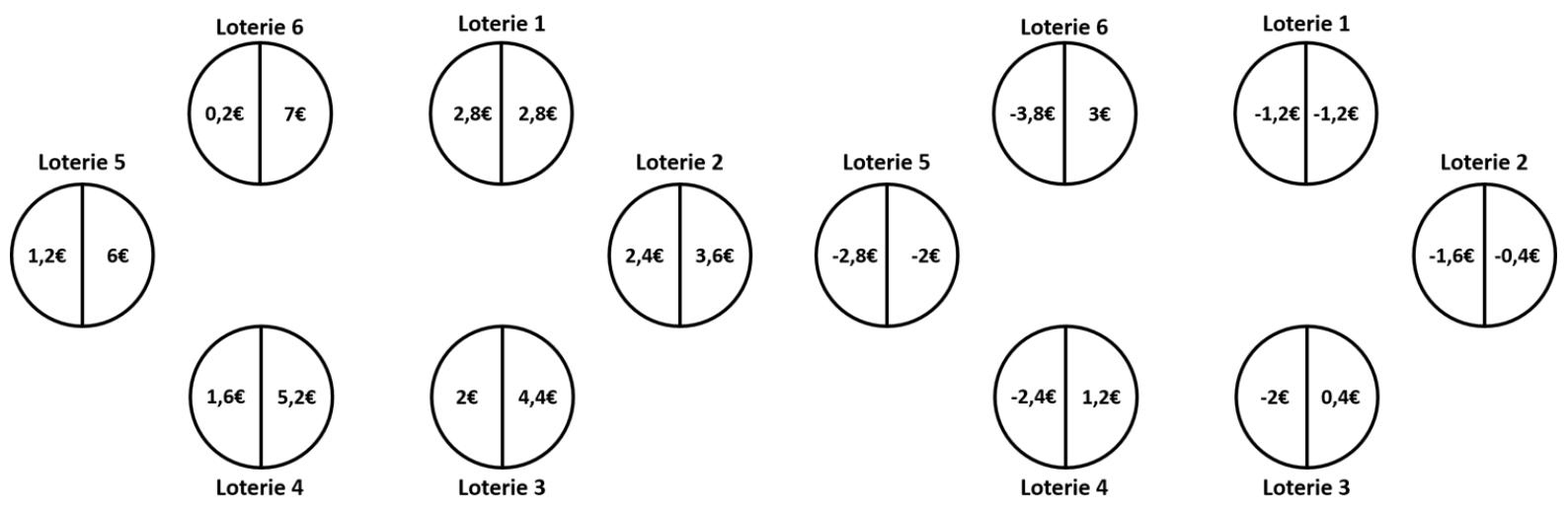

Figure B.2: Screenshot of the lotteries in the online questionnaire. 


\section{Demographic characteristics}

Table C.1: Demographic characteristics.

\begin{tabular}{lccccc}
\hline \hline Sessions & Overall & Summer & Fall & Spring & $p$-value \\
\hline Prop. Female & 0.58 & 0.56 & 0.58 & 0.60 & 0.881 \\
Prop. Student & 0.86 & 0.67 & 0.91 & 0.92 & $<0.001$ \\
Age & 23.29 & 26.91 & 21.95 & 22.49 & $<0.001$ \\
Participation & 3.65 & 6.27 & 1.92 & 4.10 & $<0.001$ \\
$N$ & 280 & 66 & 124 & 90 & - \\
\hline$N$ Session & 30 & 6 & 12 & 12 & - \\
Wage $=5$ & 10 & NoRef:2 Ref:2 & NoRef:1Ref:1 & NoRef:2 Ref:2 & - \\
Wage $=10$ & 10 & 0 & NoRef:3 Ref:3 & NoRef:2 Ref:2 & - \\
Wage $=15$ & 10 & NoRef:1 Ref:1 & NoRef:2Ref:2 & NoRef:2Ref:2 & - \\
\hline \hline
\end{tabular}

Notes: $p$-values for Kruskal-Wallis tests for continuous variables, Fisher-Exacts test for dichotomous variables. 


\section{Continuity and concavity of the utility function}

\section{D.1 Continuity}

The utility function is continuous excepted for transfers $d=\tilde{d}$ where $\tilde{d}$ is such that $\pi_{i}=\overline{\pi_{i}}$ and $\pi_{j}=\overline{\pi_{j}}$. For this value, it is yet to be proven.

$$
\begin{aligned}
& \lim _{d \rightarrow \tilde{d}^{-}} u=m\left(\overline{\pi_{i}}\right)+\alpha m\left(\overline{\pi_{j}}\right)+\lambda\left(m\left(\pi_{i}\right)-m\left(\overline{\pi_{i}}\right)\right)^{\beta}+\alpha \lambda\left(m\left(\pi_{j}\right)-m\left(\overline{\pi_{j}}\right)\right)^{\beta} \\
& \lim _{d \rightarrow \tilde{d}^{+}} u=m\left(\overline{\pi_{i}}\right)+\alpha m\left(\overline{\pi_{j}}\right)+\left(m\left(\pi_{i}\right)-m\left(\overline{\pi_{i}}\right)\right)^{\gamma}+\alpha\left(m\left(\pi_{j}\right)-m\left(\overline{\pi_{j}}\right)\right)^{\gamma}
\end{aligned}
$$

It is easy to see that $\lim _{d \rightarrow \tilde{d}-} u=\lim _{d \rightarrow \tilde{d}+} u: u$ is continuous at $d=\tilde{d}$.

\section{D.2 Concavity}

The concavity of the utility function in the gain domain directly follows from the concavity of $m()$ and $\mu()$ in the gain domain. In the loss domain, $\mu()$ is convex, but for values of $\beta$ close to one, $u$ is still concave. To show it, we study the sign of the second derivative of the utility function with respect to $\pi_{i}$ in the loss domain, depicted in Equation D.1:

$$
\begin{aligned}
u^{\prime \prime} & =m^{\prime \prime}\left(\pi_{i}\right)+\alpha m^{\prime \prime}\left(\pi_{j}\right)+\lambda \beta m^{\prime \prime}\left(\pi_{i}\right)\left(-\left(m(\pi)-m\left(\overline{\pi_{i}}\right)\right)^{\beta-1}\right. \\
& +\lambda \beta(1-\beta) m^{\prime}\left(\pi_{i}\right)\left(-\left(m\left(\pi_{i}\right)-m\left(\overline{\pi_{i}}\right)\right)^{\beta-2}\right. \\
& +\lambda \alpha \beta m^{\prime \prime}\left(\pi_{j}\right)\left(-\left(\alpha m\left(\pi_{j}\right)-\alpha m\left(\bar{\pi}_{j}\right)\right)^{\beta-1}\right. \\
& +\lambda \alpha \beta(1-\beta) \beta m^{\prime}\left(\pi_{j}\right)\left(-\left(\alpha\left(m\left(\pi_{j}\right)-\alpha m\left(\overline{\pi_{j}}\right)\right)^{\beta-2}\right.\right.
\end{aligned}
$$

For values of $\beta$ close to 1 , this expression is negative, and $u$ is concave. Such values of $\beta$ denote a low diminishing sensitivity in the loss domain and corresponds to the empirical measures. For an overview of the estimates of $\beta$ in the literature, see Fox and Poldrack (2014). 


\section{E Details of the calculation for Proofs $1 \& 2$}

Calculation for Proof 1 . We must check that the following condition holds.

$$
\begin{aligned}
& \lambda \beta m^{\prime}\left(\bar{w}+\overline{d_{\bar{w}}} E\right)\left(m\left(\overline{\pi_{i}}\right)-m\left(\bar{w}+\overline{d_{\bar{w}}}\right) E\right)^{\beta-1} \\
& -\lambda \beta \alpha m^{\prime}\left(\bar{w}+\left(1-\overline{d_{\bar{w}}}\right) E\right)\left(\alpha m\left(\bar{w}+\left(1-\overline{d_{\bar{w}}}\right) E\right)-\alpha m\left(\overline{\pi_{j}}\right)\right)^{\beta-1}>0
\end{aligned}
$$

Since $m^{\prime}\left(\bar{w}+\overline{d_{\bar{w}}} E\right)-\alpha m^{\prime}\left(\bar{w}+\left(1-\overline{d_{\bar{w}}}\right) E\right)=0$, the former expression is positive when $\left(m\left(\overline{\pi_{i}}\right)-m\left(\bar{w}+\overline{d_{\bar{w}}}\right) E\right)^{\beta-1}>\left(\alpha m\left(\bar{w}+\left(1-\overline{d_{\bar{w}}}\right) E\right)-\alpha m\left(\overline{\pi_{j}}\right)\right)^{\beta-1}$. Given that $\beta-1<0$, it is equivalent to $\left.\left.\left(m\left(\overline{\pi_{i}}\right)-m\left(\bar{w}+\overline{d_{\bar{w}}}\right) E\right)\right)<\left(\alpha m\left(\overline{\pi_{j}}\right)-\alpha m\left(\bar{w}+\left(1-\overline{d_{\bar{w}}}\right) E\right)\right)\right)$. This can be rewritten $\left.m\left(\bar{w}+\overline{d_{\bar{w}}} E\right)-\alpha m\left(\bar{w}+\left(1-\overline{d_{\bar{w}}}\right) E\right)\right)>m\left(\overline{\pi_{i}}\right)-\alpha m\left(\overline{\pi_{j}}\right)$.

Since $\alpha<1, m^{\prime}\left(\bar{w}+\overline{d_{\bar{w}}} E\right)=\alpha m^{\prime}\left(\bar{w}+\left(1-\overline{d_{\bar{w}}}\right) E\right) \Rightarrow \bar{w}+\overline{d_{\bar{w}}} E>\bar{w}+\left(1-\overline{d_{\bar{w}}}\right) E$ and $\overline{\pi_{i}}>\overline{\pi_{j}}$. Moreover, by definition, $\left(\bar{w}+\overline{d_{\bar{w}}} E\right)-\left(\bar{w}+\left(1-\overline{d_{\bar{w}}}\right) E\right)=\overline{\pi_{i}}-\overline{\pi_{j}}$. Since $m()$ is concave, the previous implies that Condition E.1 holds.

Calculation for Proof 2. We must check that the following condition holds.

$$
\begin{aligned}
& \left.\left.\gamma m^{\prime}\left(\bar{w}+\overline{d_{\bar{w}}} E\right)\right)\left(m\left(\bar{w}+\overline{d_{\bar{w}}} E\right)\right)-m\left(\overline{\pi_{i}}\right)\right)^{\gamma-1} \\
& -\alpha \gamma m^{\prime}\left(\bar{w}+\left(1-\overline{d_{\bar{w}}}\right) E\right)\left(\alpha m\left(\bar{w}+\left(1-\overline{d_{\bar{w}}}\right) E\right)-\alpha m\left(\overline{\pi_{j}}\right)\right)^{\gamma-1}<0
\end{aligned}
$$

Since $m^{\prime}\left(\bar{w}+\overline{d_{\bar{w}}} E\right)-\alpha m^{\prime}\left(\bar{w}+\left(1-\overline{d_{\bar{w}}}\right) E\right)=0$, the former expression is negative when $\left.\left(m\left(\bar{w}+\overline{d_{\bar{w}}} E\right)\right)-m\left(\overline{\pi_{i}}\right)\right)^{\gamma-1}<\left(\alpha m\left(\bar{w}+\left(1-\overline{d_{\bar{w}}}\right) E\right)-\alpha m\left(\overline{\pi_{j}}\right)\right)^{\gamma-1}$. Given that $\gamma-1<0$, it is equivalent to $\left.m\left(\bar{w}+\overline{d_{\bar{w}}} E\right)\right)-m\left(\overline{\pi_{i}}\right)>\alpha m\left(\bar{w}+\left(1-\overline{d_{\bar{w}}}\right) E\right)-\alpha m\left(\overline{\pi_{j}}\right)$. This can be rewritten $\left.\left.m\left(\bar{w}+\overline{d_{\bar{w}}}\right) E\right)-\alpha m\left(\bar{w}+\left(1-\overline{d_{\bar{w}}}\right) E\right)\right)>m\left(\overline{\pi_{i}}\right)-\alpha m\left(\overline{\pi_{j}}\right)$.

Since $\alpha<1, m^{\prime}\left(\bar{w}+\overline{d_{\bar{w}}} E\right)=\alpha m^{\prime}\left(\bar{w}+\left(1-\overline{d_{\bar{w}}}\right) E\right) \Rightarrow \bar{w}+\overline{d_{\bar{w}}} E>\bar{w}+\left(1-\overline{d_{\bar{w}}}\right) E$ and $\overline{\pi_{i}}>\overline{\pi_{j}}$. Moreover, by definition, $\left(\bar{w}+\overline{d_{\bar{w}}} E\right)-\left(\bar{w}+\left(1-\overline{d_{\bar{w}}}\right) E\right)=\overline{\pi_{i}}-\overline{\pi_{j}}$. Since $m()$ is concave, the previous implies that Condition E.2 holds. 


\section{F Supplementary regressions.}

Table F.1: Treatment effects over the different levels of wage, depending on whether the dictator is loss-averse.

\begin{tabular}{lcccccc}
\hline \hline & $(1)$ & $(2)$ & $(3)$ & $(4)$ & $(5)$ & $(6)$ \\
& Transfer & Transfer & Transfer & Transfer=0 & Transfer=0 & Transfer=0 \\
\hline Treatment effect & & & & & & \\
Wage=5 Not loss averse & -0.389 & -0.266 & -0.133 & $0.145^{*}$ & $0.122^{*}$ & 0.088 \\
& $(0.285)$ & $(0.306)$ & $(0.293)$ & $(0.076)$ & $(0.070)$ & $(0.060)$ \\
Wage=5 Loss averse & $-1.276^{* *}$ & $-1.138^{*}$ & $-1.151^{* *}$ & 0.215 & 0.199 & $0.232^{* *}$ \\
& $(0.646)$ & $(0.670)$ & $(0.485)$ & $(0.158)$ & $(0.165)$ & $(0.113)$ \\
Wage=10 Not loss averse & -0.060 & -0.163 & -0.057 & 0.008 & 0.027 & -0.010 \\
& $(0.500)$ & $(0.467)$ & $(0.457)$ & $(0.089)$ & $(0.081)$ & $(0.085)$ \\
Wage=10 Loss averse & -1.013 & -1.00 & -0.881 & 0.161 & 0.158 & 0.103 \\
& $(0.644)$ & $(0.667)$ & $(0.724)$ & $(0.171)$ & $(0.179)$ & $(0.167)$ \\
Wage=15 Not loss averse & 0.284 & 0.340 & 0.355 & -0.103 & -0.112 & -0.116 \\
& $(0.379)$ & $(0.365)$ & $(0.396)$ & $(0.094)$ & $(0.081)$ & $(0.081)$ \\
Wage=15 Loss averse & 0.664 & 0.811 & 0.797 & $-0.173^{*}$ & $-0.209^{*}$ & $0.213^{*}$ \\
& $(0.618)$ & $(0.672)$ & $(0.648)$ & $(0.101)$ & $(0.112)$ & $(0.112)$ \\
\hline Individual Char. & No & Yes & Yes & No & Yes & Yes \\
Month F.E. & No & No & Yes & No & No & Yes \\
\hline Observations & 280 & 280 & 280 & 280 & 280 & 280 \\
\hline \hline
\end{tabular}

Notes: Robust standard errors clustered at the session level are in parentheses. (1)-(3) are Tobit models. (4)-(6) are Logit models. Marginal effects reported. Treatment effect corresponds to the marginal effect of the Ref condition for loss averse and non loss averse participants. ${ }^{*} p<0.10,{ }^{* *} p<0.05,{ }^{* * *}$ $p<0.01,{ }^{* * * *} p<0.001$. 


\section{G Analysis of the self-reported emotion data.}
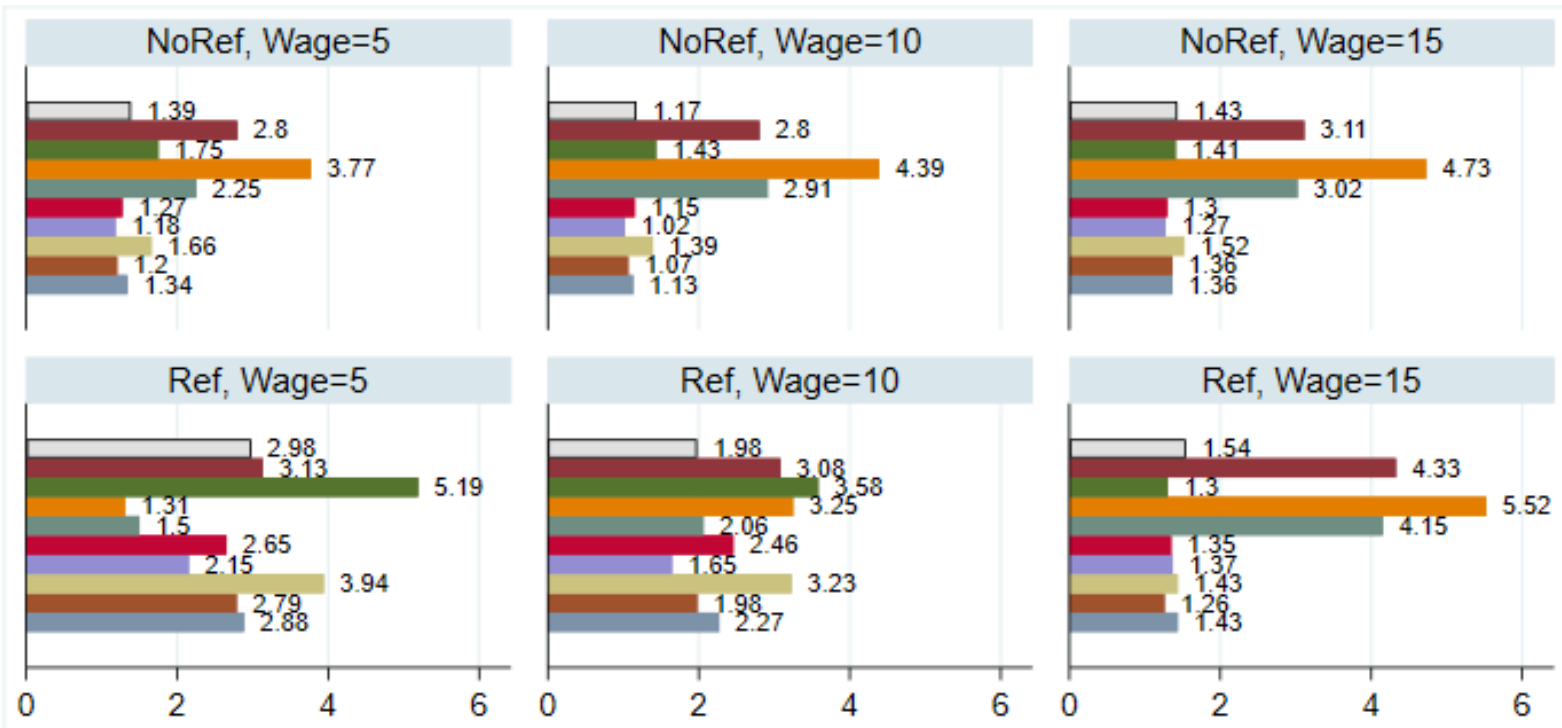

Self-reported value

\begin{tabular}{|l|l|}
\hline$\square$ anger & surprise \\
disappointement & joy \\
elation & jealousy \\
rage & frustration \\
irritation & sadness \\
\hline
\end{tabular}

Graphs by TRAITEMENT and Wage

Figure G.1: Average responses for each items of the emotion questionnaire, by condition

Table G.1 gives the pairwise correlations between the responses to the 10 items of the emotion questionnaire. We ran a factor analysis to check whether the questionnaire can be reduced to a small set of factors. This analysis confirms that two factors capture most of the variation in the responses. The first factors is best explained by the negative emotions: Anger, Disappointment, Jealousy, Rage, Frustration, Irritation and Sadness. The second factor is best explained by the positive emotions: Joy, Elation and Surprise.

Table G.1: Pairwise correlations between responses in the emotion questionnaire.

\begin{tabular}{|c|c|c|c|c|c|c|c|c|c|c|}
\hline & Anger & Surprise & Disap. & Joy & Elation & Jealousy & Rage & Frust. & Irrit. & Sad. \\
\hline Anger & 1 & & & & & & & & & \\
\hline Surprise & $0.184^{* * *}$ & 1 & & & & & & & & \\
\hline Disappointment & $0.615^{* * *}$ & 0.042 & 1 & & & & & & & \\
\hline Joy & $-0.238^{* * *}$ & $0.403^{* * *}$ & $-0.536^{* * *}$ & 1 & & & & & & \\
\hline Elation & -0.080 & $0.379^{* * *}$ & $-0.276^{* * *}$ & $0.655^{* * *}$ & 1 & & & & & \\
\hline Jealousy & $0.536^{* * *}$ & 0.091 & $0.595^{* * *}$ & $-0.209^{* * *}$ & $-0.107^{*}$ & 1 & & & & \\
\hline Rage & $0.663^{* * *}$ & $0.100^{*}$ & $0.585^{* * *}$ & $-0.175^{* * *}$ & -0.007 & $0.595^{* * *}$ & 1 & & & \\
\hline Frustration & $0.582^{* * *}$ & 0.040 & $0.780^{* * *}$ & $-0.385^{* * *}$ & $-0.197^{* * *}$ & $0.641^{* * *}$ & $0.585^{* * *}$ & 1 & & \\
\hline Irritation & $0.758^{* * *}$ & 0.057 & $0.630^{* * * *}$ & $-0.273^{* * *}$ & $-0.133^{* *}$ & $0.614^{* * *}$ & $0.714^{* * *}$ & $0.718^{* * *}$ & 1 & \\
\hline Sadness & $0.663^{* * *}$ & 0.091 & $0.660^{* * * *}$ & $-0.238^{* * *}$ & -0.087 & $0.596^{* * *}$ & $0.726^{* * *}$ & $0.652^{* * *}$ & $0.764^{* * *}$ & 1 \\
\hline
\end{tabular}

Notes: $N=280,{ }^{*} p<0.10,{ }^{* *} p<0.05,{ }^{* * *} p<0.01$, Bonferroni corrections for multiple comparisons are used. 


\section{H Analysis of the physiological data.}

Skin conductance (SC) is composed of two activities, a tonic one that corresponds to individuals' baseline activity and a phasic one that corresponds to individuals' SC activity associated with an occurring event. In our case we are interested in our participants' phasic activity around the revelation of the wage level, in the Ref conditions, or at the reminder of the wage level in the NoRef conditions. The time windows used for our analysis was 2 seconds before the display of the wage level up to 10 seconds after in the Ref conditions. In the NoRef conditions, we considered the signal at the time of the reminder up to 12 seconds after. Then, to extract the phasic activity, we treated our SC data with Ledalab (www.ledalab.de, Version: 3.4.9). This program applies a continuous de-convolution of the SC signal that disentangle phasic and tonic activities (Benedek and Kaernbach, 2010). The default parameters in Ledalab were used to treat our data. The outcome of such computation gives the integrated SCRs (ISCRs, unit: $\mu$ Siemens $(S \mu)$ ) which are the time integrals of the phasic drivers corresponding the emotional arousal induced by the events taking place during a 12 seconds time window. Following the work of Sokol-Hessner et al. (2009) and Ring and Schmidt (2019) we used the square-root of the ISCRs $(\sqrt{\mu S})$ as our variable of interest for our analysis to reduce the skewness of the raw data. In the Ref conditions when considering the anticipatory reaction we extracted the ISCRs signal from a time windows spamming from 2 seconds before the revelation of the wage level up to 2 seconds after. The remaining time windows $(+2$ to +10 seconds after the wage revelation) in considered to be specific to the emotional arousal induced by the wage revelation itself.

Table H.1: Anticipatory and wage revelation ISCRs in the Ref conditions

\begin{tabular}{lccc}
\hline \hline & Wage=5 & Wage=10 & Wage=15 \\
\hline \hline ISCR Anticipatory & $1.81(0.96)$ & $1.94(0.91)$ & $1.55(0.89)$ \\
ISCR Reveal & $1.39(0.81)$ & $1.47(1.63)$ & $1.56(0.84)$ \\
\hline p-value & $<0.001$ & $<0.001$ & 0.755 \\
\hline
\end{tabular}

Notes: Standard deviation in parentheses. $p$-values for two-sided Signed-rank tests. 


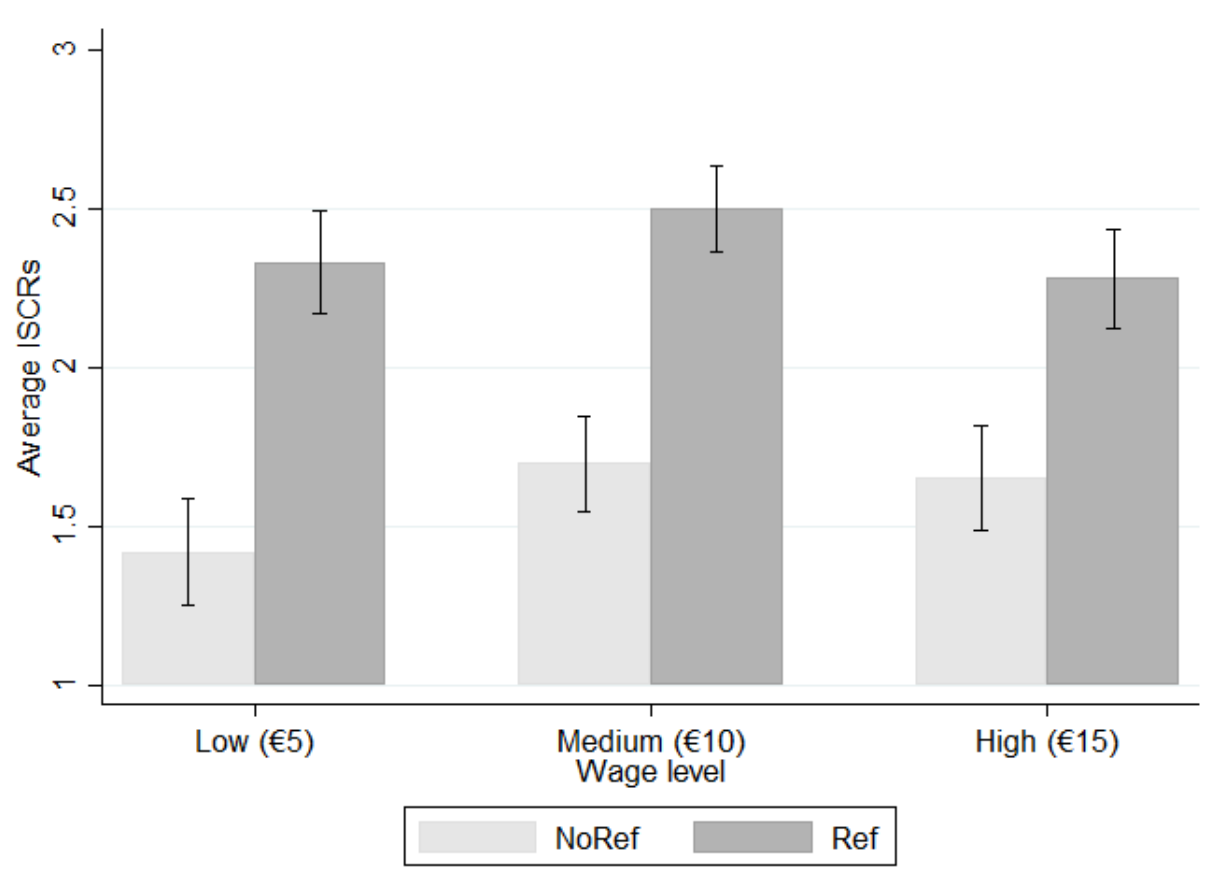

Figure H.1: Electrodermal responses around the wage revelation $(R e f)$ or at the reminder (NoRef) by experimental condition.

Table H.2: The effect of high anticipatory emotional arousal over the different levels of wage for the Ref treatment.

\begin{tabular}{lcccccc}
\hline \hline & $(1)$ & $(2)$ & $(3)$ & $(4)$ & $(5)$ & $(6)$ \\
& Transfer & Transfer & Transfer & Transfer=0 & Transfer=0 & $\begin{array}{c}\text { Transfer=0 } \\
\text { Effect of high emotional arousal at: }\end{array}$ \\
Wage=5 & $-2.014^{*}$ & $-2.0 .37^{* *}$ & $-2.160^{* *}$ & $0.385^{* *}$ & $0.398^{* *}$ & $0.414^{* * *}$ \\
& $(1.058)$ & $(0.991)$ & $(0.977)$ & $(0.191)$ & $(0.177)$ & $(0.152)$ \\
Wage=10 & -0.637 & -0.636 & -0.624 & 0.083 & 0.090 & 0.060 \\
& $(0.687)$ & $(0.644)$ & $(0.678)$ & $(0.190)$ & $(0.180)$ & $(0.186)$ \\
Wage=15 & -0.095 & -0.020 & -0.077 & -0.043 & -0.048 & -0.030 \\
& $(0.339)$ & $(0.315)$ & $(0.302)$ & $(0.068)$ & $(0.052)$ & $(0.051)$ \\
\hline Individual Char. & No & Yes & Yes & No & Yes & Yes \\
Month F.E. & No & No & Yes & No & No & Yes \\
\hline Observations & 146 & 146 & 146 & 146 & 146 & 146 \\
\hline \hline
\end{tabular}

Notes: Robust standard errors clustered at the session level are in parentheses. (1)-(3) are Tobit models. (4)-(6) are Logit models. Marginal effects reported. ${ }^{*} p<0.10,{ }^{* *} p<0.05,{ }^{* * *} p<0.01$. 
Table H.3: The effect of high emotional arousal at the time of the wage revelation over the different levels of wage for the Ref treatment.

\begin{tabular}{lcccccc}
\hline \hline & $\begin{array}{c}(1) \\
\text { Transfer }\end{array}$ & $\begin{array}{c}(2) \\
\text { Transfer }\end{array}$ & $\begin{array}{c}(3) \\
\text { Transfer }\end{array}$ & $\begin{array}{c}(4) \\
\text { Transfer=0 }\end{array}$ & $\begin{array}{c}(5) \\
\text { Transfer=0 }\end{array}$ & $\begin{array}{c}(6) \\
\text { Transfer=0 }\end{array}$ \\
\hline Effect of high emotional arousal at: & & & & & & 0.141 \\
Wage $=5$ & -0.771 & -0.917 & -0.911 & 0.0769 & 0.111 & $(0.126)$ \\
& $(0.681)$ & $(0.729)$ & $(0.764)$ & $(0.131)$ & $(0.132)$ & \\
Wage $=10$ & 0.120 & 0.286 & 0.268 & -0.0833 & -0.0972 & -0.0944 \\
& $(0.799)$ & $(0.735)$ & $(0.786)$ & $(0.228)$ & $(0.210)$ & $(0.214)$ \\
Wage=15 & 0.249 & 0.222 & 0.265 & $-0.130^{* * *}$ & -0.101 & -0.090 \\
& $(0.305)$ & $(0.320)$ & $(0.329)$ & $(0.045)$ & $(0.071)$ & $(0.068))$ \\
\hline Individual Char. & No & Yes & Yes & No & Yes & Yes \\
Month F.E. & No & No & Yes & No & No & Yes \\
\hline Observations & 146 & 146 & 146 & 146 & 146 & 146 \\
\hline \hline
\end{tabular}

Notes: Robust standard errors clustered at the session level are in parentheses. (1)-(3) are Tobit models. (4)-(6) are Logit models. Marginal effects reported. ${ }^{*} p<0.10,{ }^{* *} p<0.05,{ }^{* * *} p<0.01$. 